\title{
Joint Optimization Schemes for Cooperative Wireless Information and Power Transfer Over Rician Channels
}

Deepak Mishra, Swades De and Carla-Fabiana Chiasserini

The self-archived postprint version of this journal article is available at Linköping University Institutional Repository (DiVA):

http:/ / urn.kb.se/ resolve?urn=urn:nbn:se:liu:diva-155758

N.B.: When citing this work, cite the original publication.

Mishra, D., De, S., Chiasserini, C., (2016), J oint Optimization Schemes for Cooperative Wireless Information and Power Transfer Over Rician Channels, IEEE Transactions on Communications, 64(2), 554-571. https:// doi.org/ 10.1109/TCOMM.2015.2506699

Original publication available at:

https:/ / doi.org/ 10.1109/TCOMM.2015.2506699

Copyright: Institute of Electrical and Electronics Engineers (IEEE)

http:// www.ieee.org/ index.html

(C)2016 IEEE. Personal use of this material is permitted. However, permission to reprint/republish this material for advertising or promotional purposes or for creating new collective works for resale or redistribution to servers or lists, or to reuse any copyrighted component of this work in other works must be obtained from the IEEE. 


\title{
Joint Optimization Schemes for Cooperative Wireless Information and Power Transfer over Rician Channels
}

\author{
Deepak Mishra, Swades De, and Carla-Fabiana Chiasserini
}

\begin{abstract}
Simultaneous wireless information and power transfer (SWIPT) can lead to uninterrupted network operation by integrating radio frequency ( $R F$ ) energy harvesting with data communication. In this paper, we consider a two-hop sourcerelay-destination network and investigate the efficient usage of a decode-and-forward (DF) relay for SWIPT toward the energyconstrained destination. In particular, by assuming a Rician fading environment, we jointly optimize power allocation (PA), relay placement (RP), and power splitting (PS) so as to minimize outage probability under the harvested power constraint at the destination node. We consider the two possible cases of source-to-destination distance: (i) small distance with direct information transfer link; and (ii) relatively large distance with no direct reachability. Analytical expressions for individual and joint optimal PA, RP, and PS are obtained by exploiting convexity of outage minimization problem for the no direct link case. In case of direct source-to-destination link, multi-pseudoconvexity of joint-optimal PA, RP, and PS problem is proved, and alternating optimization is used to find the global optimal solution. Numerical results show that the joint optimal solutions, although strongly influenced by the harvested power requirement at the destination, can provide respectively $64 \%$ and $100 \%$ outage improvement over the fixed allocation scheme for without and with direct link.

Index Terms-RF energy harvesting; Rician fading; decode and forward; power allocation; relay placement; power splitting ratio; outage-harvested power tradeoff; alternating optimization.
\end{abstract}

\section{INTRODUCTION AND BACKGROUND}

Relay-assisted data communication and cooperative transmission strategies offer significant benefits over the direct source-to-destination transmission. The advantages include cooperative diversity, energy saving, increased secrecy, network coverage extension, and improvement of quality-of-service in wireless networks. Moreover, cooperative relaying techniques can overcome high path-loss, blocking or shadowing losses, and high transmit power requirements, by providing alternate path(s) from source to destination via one or more relays. There are several studies on optimal power allocation (PA) and relay placement (RP) for cooperative amplify-and-forward (AF) as well as decode-and-forward (DF) information relaying under different fading conditions [1]-[6]. Minimization of source-sum-power subject to outage constraints using DF relay

D. Mishra and S. De are with the Department of Electrical Engineering and Bharti School of Telecommunication, Indian Institute of Technology Delhi, New Delhi, India (e-mail: \{deepak.mishra, swadesd\}@ee.iitd.ac.in). C.-F Chiasserini is with Politecnico di Torino, Torino, Italy, and is also a Research Associate with the Institute of Electronics, Computer and Telecommunication Engineering of the National Research Council of Italy (IEIIT-CNR), Torino, Italy (e-mail: chiasserini@polito.it).

This work has been supported by the Department of Science and Technology (DST) under the grant no. SB/S3/EECE/0248/2014. is studied in [7] and [8], respectively with as well as without direct links between multiple sources and single destination.

Another line of research that has recently emerged is radio frequency (RF) energy harvesting (RFEH) at the energyconstrained field nodes, which can prolong the lifetime of wireless networks. Since most of the long-range communication is based on transmission of RF signals, usage of this $\mathrm{RF}$ radiation for energy harvesting leads to simultaneous wireless information and power transfer (SWIPT) to the energyconstrained receiver. SWIPT is discussed in the pioneering works [9], [10]. The study in [11] introduces two mechanisms for practical implementation of SWIPT: a) power splitting (PS) and b) time switching (TS). Subsequently, PS-based and TSbased routing protocols for RFEH AF relay node and single source-destination $(\mathcal{S}-\mathcal{D})$ pair are proposed in [12]. A dualhop RFEH AF relaying system, with and without the presence of co-channel interference is investigated in [13]. PA strategies for RFEH DF relay for multiple $\mathcal{S}-\mathcal{D}$ pairs are proposed in [14]. The performance of a dual-hop RFEH full-duplex relaying system is studied in [15] for both AF and DF relaying protocols. Authors in [15], also investigated optimal TS ratio under different communication modes. SWIPT without as well as with cooperative energy relaying is discussed in [16], and the impact of spatial randomness of relay locations on the performance of SWIPT is studied in [17]. The work in [18] demonstrated that there exists a trade-off between information and energy transfer for relay selection in SWIPT, as the preferable relay position is different for information transfer and energy transfer. Yet, optimal PA and RP are not considered in [12]-[18]. It is worth noting that [12]-[15] consider source-relay and relay-destination distances as constants, whereas [16]-[18] consider relay selection strategies. Also, the optimal PA and RP problem investigated in [1]-[8] for twohop information relaying, do not consider the Rician fading model, which is more appropriate to incorporate the effect of strong line-of-sight (LOS) component in SWIPT [19], [20] and information relaying systems [21].

Accounting for the system and wireless device constraints, it is argued in [22] that multi-hop RF energy transfer can improve RFEH efficiency by deploying relay nodes close to the target energy receiver. In this technique, the relay first collects the otherwise-dispersed RF energy of the source and then transfers it to the energy receiver, which reduces path loss and improves RF-to-DC conversion efficiency due to a higher received power [23]. Two-hop RF energy transfer and multipath energy routing have been experimentally demonstrated 
TABLE I: Joint cooperative optimization schemes for SWIPT.

\begin{tabular}{|c|c|c|}
\hline $\begin{array}{c}\text { Optimization } \\
\text { scheme }\end{array}$ & Practical setting & $\begin{array}{c}\text { Node(s) where } \\
\text { optimization } \\
\text { is performed }\end{array}$ \\
\hline Optimal PA & $\begin{array}{c}\mathcal{S} \text { and } \mathcal{R} \text {, connected to the common power grid } \\
\text { or having common energy resource, cooperate } \\
\text { to share the total power budget optimally }\end{array}$ & $\mathcal{S}$ or $\mathcal{R}$ \\
\hline Optimal RP & $\begin{array}{c}\text { When there are no terrain asperities or blockage, } \\
\mathcal{R} \text { can adjust, or can be instructed by } \mathcal{S} \text { to adjust, } \\
\text { its position optimally to aid efficient SWIPT to } \mathcal{D}\end{array}$ & $\mathcal{R}$ or $\mathcal{S}$ \\
\hline Optimal PS & $\begin{array}{c}\text { RFEH } \mathcal{D} \text { has enough energy resources } \\
\text { to carry out the PS optimization }\end{array}$ & $\mathcal{D}$ \\
\hline Joint-optimal & $\begin{array}{c}\text { It has the luxury of combining the merits } \\
\text { of all three optimization schemes }\end{array}$ & $\mathcal{S}, \mathcal{R}$, and $\mathcal{D}$ \\
\hline
\end{tabular}

recently in [23] and [24]. These works however have not looked into joint information and RF energy transfer aspects. Intuitively, optimal PA and RP in SWIPT are quite different from those in conventional information transfer [1]-[8], where RF energy transfer using relay is not considered.

In this paper we study the outage performance of a two-hop, half-duplex DF relay-assisted SWIPT with a single source $\mathcal{S}$ and destination $\mathcal{D}$. We consider the two possible cases: (i) relatively short $\mathcal{S}$-to- $\mathcal{D}$ distance where $\mathcal{D}$ is capable of receiving information directly from $\mathcal{S}$; and (ii) long $\mathcal{S}$-to- $\mathcal{D}$ distance, with no direct communication link [2]-[6]. However, in both cases $\mathcal{D}$ is not capable of harvesting energy from $\mathcal{S}$ due to low RF energy transfer range [23]. $\mathcal{S}$ and relay $\mathcal{R}$ are assumed to have enough energy resources, whereas $\mathcal{D}$ operates with the harvested RF energy from the received signal from $\mathcal{R}$ using the PS technique. To improve the efficiency of DF relay-assisted SWIPT, we propose four different optimization schemes under varying real-world constraints (practical settings), as mentioned in Table I. The table also underlines at which node(s) the optimization is performed. The practical settings for the problem considered include self-sustainable broadcasting networks and multiuser downlink SWIPT systems, where the user devices are battery constrained, whereas the broadcasting base station and the relay are connected to the power grid [18]. $\mathcal{S}$ and $\mathcal{R}$ can also be considered as infrastructure nodes in network-assisted device-to-device (D2D) communications or Long-Term Evolution (LTE) Advanced system, which share the total power for efficient information and energy transfer to the nearby battery-constrained wireless devices.

To the best of our knowledge, this is the first work that presents a joint optimization of PA, RP, and PS for SWIPT to minimize the outage probability at $\mathcal{D}$ without and with direct communication link from $\mathcal{S}$. To incorporate the effect of strong LOS component in SWIPT, outage performance analysis is done using Rician fading model, which has not been considered before. While minimizing outage probability $p_{\text {out }}$, we consider constraints on total transmit power $P_{T}$ (sum of $\mathcal{S}$ and $\mathcal{R}$ power) and required harvested power $\zeta_{P}$ at $\mathcal{D}$. Our key contributions are as follows.

- Joint optimization schemes for cooperative SWIPT to enhance outage performance of $\mathcal{R}$-assisted $\mathcal{S}$-to- $\mathcal{D}$ communication are presented for both without and with $\mathcal{S}$-to$\mathcal{D}$ direct link. All optimization results are derived under practical RFEH constraints at $\mathcal{D}$, while considering the Rician channel fading to incorporate the dominant LOS component of the links. The results for Rayleigh fading can be easily generated by setting the Rice factor as zero.
- In SWIPT with no $\mathcal{S}$-to- $\mathcal{D}$ link, analytical expressions are obtained for both individual and joint-optimal PA, RP, and PS to minimize $p_{\text {out }}$, subject to $P_{T}$ and $\zeta_{P}$ constraints.

- For short $\mathcal{S}$-to- $\mathcal{D}$ distance with direct communication link between $\mathcal{S}$ and $\mathcal{D}$, tri-pseudoconvexity of $p_{\text {out }}$ is proved. Subsequently, for individual PA, RP, and PS optimization, semi-closed-form solutions are obtained by exploiting individual pseudoconvexity of $p_{\text {out }}$ for each problem. The joint-optimal solution is obtained by using alternating optimization technique along with bi-pseudoconvexity of $p_{\text {out }}$ with optimized PS in $P_{s}$ and $d$.

- Impact of RFEH requirement at $\mathcal{D}$ on optimal PA, RP, and PS for efficient SWIPT is discussed via numerical results. Improved performance of the proposed joint and individual optimization schemes over non-cooperative fixed allocation scheme is also demonstrated. For example, with respect to fixed allocation scheme, joint optimization offers about $64 \%$ and $100 \%$ improvement in $p_{\text {out }}$ for without and with direct $\mathcal{S}$-to- $\mathcal{D}$ link, respectively.

- Trade-off between $p_{\text {out }}$ and $\zeta_{P}$ is investigated in the proposed joint optimization scheme under different Rice factor values. The impacts of transmit power budget, $\mathcal{S}$-to- $\mathcal{D}$ distance, and channel conditions on optimized solutions and minimized $p_{\text {out }}$ are also studied.

The rest of the paper is organized as follows. Network topology considered and its motivation are discussed in Section II. Problem definition is presented in Section III. Optimal PA for fixed RP and PS, without and with direct $\mathcal{S}$-to- $\mathcal{D}$ link availability is presented in Section IV. Section V contains analytical solutions for optimal RP with predetermined PA and PS for both short and long $\mathcal{S}$-to- $\mathcal{D}$ distance cases. PS ratio optimization is studied in Section VI. Joint-optimal PA, RP, and PS scheme, exploiting convexity and multi-pseudoconvexity of $p_{\text {out }}$ respectively for no direct link and with $\mathcal{S}$-to- $\mathcal{D}$ direct link, is analyzed in Section VII. Numerical results are presented in Section VIII, followed by concluding remarks in Section IX.

\section{SYSTEM MODEL}

Here we discuss the network and channel models along with motivation for these consideration.

\section{A. Network topology and channel model}

We consider a three-node, two-hop wireless network, consisting of an information source $\mathcal{S}$, a relay node $\mathcal{R}$, and a destination node $\mathcal{D}$ placed on a two-dimensional Euclidean plane. We consider two system models for RP, depending on the availability of direct $\mathcal{S}$-to- $\mathcal{D}$ communication link: linear and elliptical. In the first case (Fig. 1(a)), when $D$ is reasonably large, there is no direct $\mathcal{S}$-to- $\mathcal{D}$ link available due to large path loss, shadowing, and fading effects. Hence, here $\mathcal{R}$ is placed on the LOS path between $\mathcal{S}$ and $\mathcal{D}$ to maximize the gain from relaying. In the second case, with direct $\mathcal{S}$-to- $\mathcal{D}$ link availability (Fig. 1(b)), $\mathcal{R}$ is placed at a position along the locus of the ellipse [6], [25] to avoid the obstruction to direct $\mathcal{S}$-to- $\mathcal{D}$ link. $\mathcal{S}$ and $\mathcal{D}$, separated by a distance $D$, are located at the two foci of the ellipse.

$\mathcal{R}$ operates in half-duplex DF mode. Thus, the information transfer occurs in two slots: in the first slot from $\mathcal{S}$ to $\mathcal{R}$ (and 


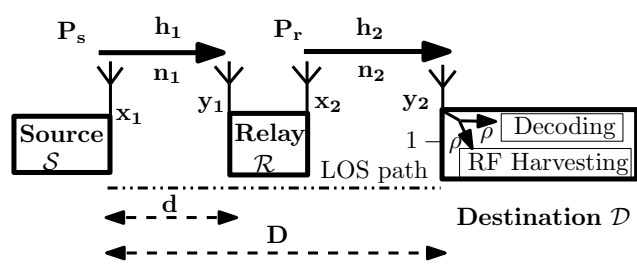

(a) Relay placement on linear LOS path for large $\mathcal{S}$-to- $\mathcal{D}$ distance with no direct $\mathcal{S}$-to- $\mathcal{D}$ link

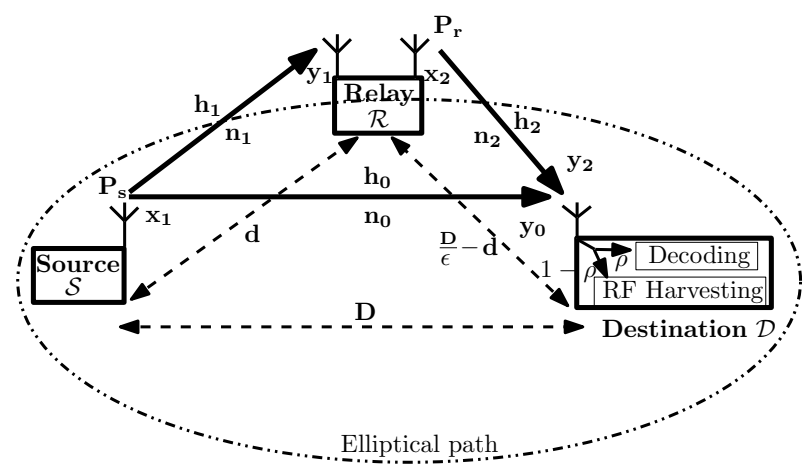

(b) Relay placement on elliptical path for short $\mathcal{S}$-to- $\mathcal{D}$ distance with direct $\mathcal{S}$-to- $\mathcal{D}$ link availability

Fig. 1: Three-node network topology considering two $\mathcal{S}$-to- $\mathcal{D}$ distance-based cases with $\mathcal{R}$ having two directional antennas.

$\mathcal{S}$ to $\mathcal{D}$ if direct $\mathcal{S}$-to- $\mathcal{D}$ link is available), and in the second slot from $\mathcal{R}$ to $\mathcal{D}$. It may be noted that, although the intended half-duplex operation could be conducted using single omnidirectional antenna at each node as in conventional cooperative communication systems, we consider two directional antennas at $\mathcal{R}$ (Fig. 1). One is directed towards $\mathcal{D}$ - essentially for efficient $\mathcal{R}$-to- $\mathcal{D}$ energy transfer (or SWIPT), and the other is directed towards $\mathcal{S}$ for effective $\mathcal{S}$-to- $\mathcal{R}$ information transfer.

Indeed, $\mathcal{D}$ has RFEH capability. The RFEH operation is based on PS technique [11], in which the received power is split into two parts with a PS ratio $\rho \in(0,1)$. A fraction $\rho$ of the received power at $\mathcal{D}$ is used for data detection or decoding, and the remaining fraction $(1-\rho)$ is used for RFEH. For simplicity, an ideal PS is assumed, neglecting the power loss, noise degradation, and synchronization errors. The received signal $y_{0}$ at $\mathcal{D}$ and $y_{1}$ at $\mathcal{R}$ from $\mathcal{S}$ in the first slot, and $y_{2}$ at $\mathcal{D}$ from $\mathcal{R}$ in the second slot are given by:

$y_{0}=h_{0} \sqrt{P_{s}} x_{1}+n_{0}, \quad y_{1}=h_{1} \sqrt{P_{s}} x_{1}+n_{1}, \quad y_{2}=h_{2} \sqrt{P_{r}} x_{2}+n_{2}$

where $n_{0}, n_{1}$, and $n_{2}$ are mutually independent Additive White Gaussian Noise at the respective receivers, with zero mean and same noise power $N_{0} . P_{s}$ and $P_{r}$ are the transmit powers of $\mathcal{S}$ and $\mathcal{R}$, respectively, with $P_{T}=P_{s}+P_{r}$ as the total transmit power budget. $x_{1}$ and $x_{2}$ are the signals transmitted by $\mathcal{S}$ and $\mathcal{R}$, respectively. We also assume that $\mathbb{E}\left[x_{i}\right]=0$ and $\mathbb{E}\left[\left|x_{i}\right|^{2}\right]=1, \forall i \in\{1,2\} . h_{0}, h_{1}$, and $h_{2}$ are the Rician channel gain coefficients. Over Rician fading channels, the instantaneous signal-to-noise ratio $(\mathrm{SNR}) \gamma_{0}$ for $\mathcal{S}$-to- $\mathcal{D}$ link, $\gamma_{1}$ for $\mathcal{S}$-to- $\mathcal{R}$ link, and $\gamma_{2}$ for $\mathcal{R}$-to- $\mathcal{D}$ link follow the weighted noncentral- $\chi^{2}$ distribution with two degrees of freedom, whose cumulative distribution function (CDF) is given by [26]:

$$
F_{\gamma_{i}}(\gamma)=1-\mathcal{C}_{\gamma_{i}}(\gamma)=1-Q_{1}\left(\sqrt{2 K_{i}}, \sqrt{\frac{2\left(K_{i}+1\right) \gamma}{\overline{\gamma_{i}}}}\right)
$$

where $\mathcal{C}_{\gamma_{i}}(\cdot)$ is the complimentary CDF of $\gamma_{i}$ and $Q_{1}(\cdot, \cdot)$ is the first order Marcum $Q$-function [26]. $K_{i}$ is the Rice factor defined as the ratio of power of LOS component to the scattered components. $\overline{\gamma_{i}}=\mathbb{E}\left[\gamma_{i}\right]$ is the average SNR of the respective links, given by: $\overline{\gamma_{0}}=\frac{a_{d} P_{s}}{N_{0} D^{l}}, \overline{\gamma_{1}}=\frac{a_{s} P_{s}}{N_{0} d^{l}}$, and $\overline{\gamma_{2}}=\frac{a_{r} P_{r}}{N_{0}\left(\frac{D}{\epsilon}-d\right)^{l}}$, where $d$ and $\left(\frac{D}{\epsilon}-d\right)$ are $\mathcal{S}$-to- $\mathcal{R}$ and $\mathcal{R}$ to- $\mathcal{D}$ distances, respectively. $\epsilon$ is the eccentricity; $\epsilon=1$ for linear case (cf. Fig. 1(a)) when there is no direct $\mathcal{S}$-to- $\mathcal{D}$ link available. $a_{d}, a_{s}$, and $a_{r}$ account for the channel parameters, namely, fading and antenna gains, in the respective link, and $l$ is the path loss exponent. The average harvested power at $\mathcal{D}$ is $P_{D}^{H}=\frac{\eta a_{r}(1-\rho) P_{r}}{\left(\frac{D}{\epsilon}-d\right)^{l}}$, where $\eta$ is the RF-to-DC conversion efficiency of the RFEH circuitry at $\mathcal{D}$.

\section{B. Motivation for proposed system model}

Our consideration of Rician fading channel model is motivated by the fact that a strong LOS component is present in practical SWIPT and information relaying scenarios with direct link availability or short communication ranges. Following this, we have employed a commonly used elliptical topology [6], [25] for RP which helps to extend the conventional line topology to a more generic two-dimensional RP model, while considering the possibility of a direct LOS path between $\mathcal{S}$ and $\mathcal{D}$. Also, it offers flexibility in realization of a realistic non-blocking model that incorporates the behavior of practical directional antennas having reduced gains with increase in angle away from the direction of main beam [27]. Hence, it allows $\mathcal{R}$ (blocking object) to come closer to $\mathcal{S}$ and $\mathcal{D}$ (transmitting and receiving directional antennas) from the perpendicular direction, yet stay far away from the main beam.

In optimal power (system resource) allocation, if independent transmit power budgets $P_{T_{\mathcal{S}}}$ and $P_{T_{\mathcal{R}}}$ are considered at $\mathcal{S}$ and $\mathcal{R}$ respectively, minimum $p_{\text {out }}$ will trivially occur at full power utilization $\left(P_{s}=P_{T_{\mathcal{S}}}, P_{r}=P_{T_{\mathcal{R}}}\right)$. Instead, we consider controlled relaying where $\mathcal{S}$ and $\mathcal{R}$ are either administered by the same service provider, or have a common energy resource that they share for efficient SWIPT to $\mathcal{D}$. One such practical setting includes $\mathcal{S}$ being a base station in a cellular scenario with $\mathcal{R}$ as a network operator controlled relay node. So, in our PA optimization, we consider a joint total transmit power budget $P_{T}=P_{s}+P_{r}$ and optimally distribute it between $\mathcal{S}$ and $\mathcal{R}$ to minimize $p_{\text {out }}$, which is also influenced by RP.

It is also worth noting that, in the proposed system model information transfer from $\mathcal{S}$-to- $\mathcal{D}$ is over two hops in addition to the possible direct $\mathcal{S}$-to- $\mathcal{D}$ communication link, whereas the energy is transferred via only one hop, from $\mathcal{R}$-to- $\mathcal{D}$. Two-hop energy transfer is not considered because of very low RFEH sensitivity [28], which leads to a very low RF energy transfer range as compared to the typical wireless data communication range [23], [24]. Hence, for a typical $\mathcal{S}$-to$\mathcal{D}$ information transfer distance and with the current state of RFEH technology [28], for practical feasibility of RFEH at $\mathcal{D}$, the transmit power $P_{s}$ at $\mathcal{S}$ has to be very large. The Effective 
Isotropic Radiated Power (EIRP) required at $\mathcal{S}$ in order to have $\zeta_{P}$ amount of DC power available after RF-to-DC conversion at $\mathcal{D}$ is given by: $\mathrm{EIRP} \triangleq P_{s} G_{s}=\frac{\zeta_{P}}{\eta(1-\rho) G_{D}}\left(\frac{4 \pi D f}{c}\right)^{l}$, where $G_{s}$ and $G_{D}$ are the antenna gains of $\mathcal{S}$ and $\mathcal{D}$, respectively, $c$ is the speed of light, and $f$ is the frequency of the transmitted signal. Considering two values of $\zeta_{P}$ as $0 \mathrm{dBm}$ and 10 $\mathrm{dBm}$ for RFEH at $\mathcal{D}$ using commercially-available Powercast $\mathrm{RF}$ harvester and antennas [29], $\rho=0.01, D=10 \mathrm{~m}$, $G_{s}=G_{D}=6.1 \mathrm{dBi}, f=915 \mathrm{MHz}$, and $l=3$, the EIRP required is at least $23.35 \mathrm{~kW}$ and $198.55 \mathrm{~kW}$, respectively. Thus, even at very low $D$, the transmit power requirements are much higher than the maximum transmit power limits defined by FCC regulations in different frequency bands. For example, at $900 \mathrm{MHz}$ band the allowable maximum EIRP is $4 \mathrm{~W}$ [30].

At last, we comment on the practical reference scenarios for the system setting considered in the paper. As noted in [9]-[19], a SWIPT-enabled network can overcome the finite lifetime limitation of battery-driven nodes, or high energy and infrastructure cost involved with the networks that are connected to the power grid. So, in order to enhance the practical applicability of SWIPT under different real-world constraints, we have proposed four optimization schemes, as mentioned in Table I. These optimization schemes can be employed individually or jointly, depending on the underlying reference scenario. For example, if we have a central controller for PA to $\mathcal{S}$ and $\mathcal{R}$, no terrain blockage for RP, and PS optimization capability at $\mathcal{D}$, all three parameters (PA, RP, and PS) can be jointly optimized. The proposed optimization is performed by the node(s) with the help of full channel state information (CSI) acquired by (i) $\mathcal{R}$ for $\mathcal{S}$-to- $\mathcal{R}$ link, (ii) $\mathcal{D}$ for $\mathcal{R}$-to- $\mathcal{D}$ link, and (iii) $\mathcal{D}$ for $\mathcal{S}$-to- $\mathcal{D}$ link, from the pilot signals sent by $\mathcal{S}, \mathcal{R}$, and $\mathcal{S}$, respectively. This collected CSI is fed back to the node which performs the optimization. Intuitively, the joint optimization scheme requires the most signaling cost due to the involvement of all three nodes, i.e., $\mathcal{S}, \mathcal{R}$, and $\mathcal{D}$, in the cooperative optimization of PA, RP, and PS to realize minimum $p_{\text {out }}$ for a given total power budget $P_{T}, \mathcal{S}$-to- $\mathcal{D}$ distance, and energy demand $\zeta_{P}$ at $\mathcal{D}$.

\section{Problem Definition}

We now derive outage probability expressions and present the proposed optimization framework.

\section{A. Outage probabilty analysis}

The outage probability $p_{\text {out }}$, a grade of service measure of the sent data, is the probability that the received signal strength falls below an information outage threshold $\zeta_{I}$. Its representation in terms of the end-to-end SNR $\gamma_{E 2 E}$ at $\mathcal{D}$ is:

$$
p_{\text {out }}=\operatorname{Pr}\left(\frac{1}{2} \log _{2}\left(1+\gamma_{E 2 E}\right)<\zeta_{I}\right) \text {. }
$$

The outage probability expressions in the two cases of $\mathcal{S}$ to- $\mathcal{D}$ reachability are obtained below.

1) No $\mathcal{S}$-to-D direct link available: Here, $\gamma_{E 2 E}$ is bottlenecked by the weaker of the two SNRs: from $\mathcal{S}$-to- $\mathcal{R}$ and from $\mathcal{R}$-to- $\mathcal{D}$ [31]. Hence, outage probability, denoted as $p_{\text {out }}$, can be represented as a function of transmit powers $\left(P_{s}, P_{r}\right)$ and the corresponding path losses as [32]:

$$
\begin{aligned}
p_{\text {out }_{1}}= & \operatorname{Pr}\left[\frac{1}{2} \log _{2}\left(1+\min \left\{\gamma_{1}, \rho \gamma_{2}\right\}\right)<\zeta_{I}\right] \\
= & \operatorname{Pr}\left[\min \left\{\gamma_{1}, \rho \gamma_{2}\right\}<2^{2 \zeta_{I}}-1\right] \\
\stackrel{\mathcal{Z} \triangleq 2^{2 \zeta_{I}}-1}{=} & 1-\left(1-\operatorname{Pr}\left[\gamma_{1}<\mathcal{Z}\right]\right)\left(1-\operatorname{Pr}\left[\gamma_{2}<\frac{\mathcal{Z}}{\rho}\right]\right) \\
= & 1-\mathcal{C}_{\gamma_{1}}(\mathcal{Z}) \mathcal{C}_{\gamma_{2}}\left(\frac{\mathcal{Z}}{\rho}\right) \\
\stackrel{\text { using (2) }}{=} & 1-Q_{1}\left(\sqrt{2 K_{1}}, \sqrt{\frac{2\left(K_{1}+1\right) N_{0} d \mathcal{Z}}{a_{s} P_{s}}}\right) \times \\
& Q_{1}\left(\sqrt{2 K_{2}}, \sqrt{\frac{2\left(K_{2}+1\right) N_{0}\left(\frac{D}{\epsilon}-d\right)^{l} \mathcal{Z}}{\rho a_{r} P_{r}}}\right) .(4)
\end{aligned}
$$

To gain analytical insights on the performance of the proposed optimization schemes for SWIPT over Rician channels, we consider a recently developed tight exponential-type approximation [33] for $Q_{1}(\cdot, \cdot)$, which is being widely considered for Rician fading performance analysis [34]:

$$
Q_{1}(a, b) \approx \exp \left(-e^{\phi(a)} b^{\varphi(a)}\right) .
$$

In above equation, the parameters $\phi(a)$ and $\varphi(a)$ are functions of $a$, and are given by:

$$
\begin{gathered}
\phi(a)= \begin{cases}\frac{45 \pi^{2}+72 \ln 2+20.7798-496}{64\left(9 \pi^{2}-80\right)} a^{4}-\frac{a^{2}}{2}-\ln 2, & a \ll 1 \\
-0.0045 a^{4}+0.0858 a^{3}-0.7529 a^{2} & \text { otherwise } \\
+0.3504 a-0.8526, & a \ll 1\end{cases} \\
\text { and } \varphi(a)= \begin{cases}\frac{9}{8\left(9 \pi^{2}-80\right)} a^{4}+2, \\
0.0053 a^{4}-0.0910 a^{3} \\
+0.5895 a^{2}-0.5916 a+2.1793, & \text { otherwise. }\end{cases}
\end{gathered}
$$

Employing the approximation (5) in (4), we obtain:

$$
p_{\text {out }_{1}} \approx 1-e^{-\left(\alpha_{1}\left(\frac{d^{l}}{a_{s} P_{s}}\right)^{\beta_{1}}+\alpha_{2}\left(\frac{(D-d)^{l}}{\rho a_{r}\left(P_{T}-P_{s}\right)}\right)^{\beta_{2}}\right)}
$$

where $\alpha_{i}=e^{\phi\left(\sqrt{2 K_{i}}\right)}\left(2\left(K_{i}+1\right) N_{0} \mathcal{Z}\right)^{\beta_{i}}$ and $\beta_{i}=\frac{\varphi\left(\sqrt{2 K_{i}}\right)}{2}$ $\forall i \in\{0,1,2\}$ are positive functions of Rice factor $K_{i}$, noise power $N_{0}$, and outage threshold $\zeta_{I}$ (as $\mathcal{Z} \triangleq 2^{2 \zeta_{I}}-1$ ). Also note that $P_{r}=P_{T}-P_{s}$. The accuracy of this exponential approximation has also been numerically verified in Section VIII-D.

2) $\mathcal{S}$-to-D direct link available: Here $\mathcal{D}$ combines signals $y_{0}$ received from $\mathcal{S}$ in first slot and $y_{2}$ received from $\mathcal{R}$ in second slot using maximal ratio combining [7]. $\gamma_{E 2 E}$ at $\mathcal{D}$ is:

$$
\gamma_{E 2 E}=\min \left\{\gamma_{1}, \gamma_{0}+\rho \gamma_{2}\right\}=\min \left\{\gamma_{1}, \Upsilon_{02}\right\}
$$

where $\Upsilon_{02}$ is the effective SNR in the second slot which is the sum of positive weighted noncentral- $\chi^{2}$ random variables. Although the distribution of this sum can be obtained in terms of Laguerre expansions [35], we consider its integral definition to avoid the unnecessary complications. Using (3) and (8), in this case the outage probability, denoted by $p_{\text {out }}$, can be represented as a function of transmit powers $\left(P_{s}, P_{r}=P_{T}-P_{s}\right)$, $\mathcal{S}$-to- $\mathcal{R}$ distance $d$, and $\rho$ as given in (9). Though the integral in (9) cannot be solved analytically, an efficient numerical solution can be easily obtained using commonly available commercial software, such as Matlab or Mathematica. 


$$
\begin{aligned}
& p_{\text {out }_{2}}=\operatorname{Pr}\left[\min \left\{\gamma_{1}, \Upsilon_{02}\right\}<2^{2 \zeta_{I}}-1\right]=1-\mathcal{C}_{\gamma_{1}}(\mathcal{Z}) \mathcal{C}_{\Upsilon_{02}}(\mathcal{Z})=1-\mathcal{C}_{\gamma_{1}}(\mathcal{Z})\left(1-\int_{0}^{\mathcal{Z}} \frac{\mathrm{d} F_{\gamma_{0}}(x)}{\mathrm{d} x} F_{\gamma_{2}}\left(\frac{\mathcal{Z}-x}{\rho}\right) \mathrm{d} x\right) \\
& \underset{\sim}{\operatorname{using}(2),(5)} 1-e^{-e^{\phi\left(\sqrt{2 K_{1}}\right)}\left(\frac{2\left(K_{1}+1\right) N_{0} \mathcal{Z} d^{l}}{a_{s} P_{s}}\right)^{\beta_{1}}}\left[1-\int_{0}^{\mathcal{Z}} \frac{\beta_{0}}{x}\left(\frac{2\left(K_{0}+1\right) N_{0} x D^{l}}{a_{d} P_{s}}\right) e^{\beta_{0}\left(\sqrt{2 K_{0}}\right)}\right. \\
& \left.\times e^{-e^{\phi\left(\sqrt{2 K_{0}}\right)}\left(\frac{2\left(K_{0}+1\right) N_{0} x D^{l}}{a_{d} P_{s}}\right)^{\beta_{0}}}\left(1-e^{-e^{\phi\left(\sqrt{2 K_{2}}\right)}\left(\frac{2\left(K_{2}+1\right) N_{0}(\mathcal{Z}-x)\left(\frac{D}{\epsilon}-d\right)^{l}}{\rho a_{r}\left(P_{T}-P_{s}\right)}\right)^{\beta_{2}}}\right) \mathrm{d} x\right] .
\end{aligned}
$$

\section{B. Optimization formulation}

Given the outage probability $p_{\text {out }}$ expressions (7) and (9) as functions of transmit powers $\left(P_{s}, P_{r}\right)$, inter-nodal distances $\left(d, \frac{D}{\epsilon}-d\right)$, and PS ratio $\rho$, we are interested in finding optimal PA for $\mathcal{S}$ and $\mathcal{R}$, optimal RP between $\mathcal{S}$ and $\mathcal{D}$, and optimal $\rho$ to minimize $p_{\text {out }}$, subject to harvested power constraint $(C 1)$, total power constraints $(C 2-C 3)$, relay placement constraints $(C 4-C 5)$, and normalization constraints on $\rho(C 6-C 7)$. The optimization problem can be formulated as:

$(\mathbf{J 0}): \underset{P_{S}, d, \rho}{\operatorname{minimize}} p_{\text {out }}= \begin{cases}p_{\text {out }_{1}}, & \text { if } \mathcal{S} \text {-to- } \mathcal{D} \text { direct link is not available } \\ p_{\text {out }_{2}}, & \text { if } \mathcal{S} \text {-to- } \mathcal{D} \text { direct link is available }\end{cases}$ subject to $C 1: P_{\text {con }}\left(P_{s}, d, \rho\right) \triangleq \zeta_{P}-\frac{\eta a_{r}(1-\rho)\left(P_{T}-P_{s}\right)}{\left(\frac{D}{\epsilon}-d\right)^{l}} \leq 0$,

$$
\begin{array}{lll}
C 2: P_{s} \leq P_{T}, & C 3: P_{s} \geq 0, & C 4: d \leq \frac{D}{\epsilon}-\delta, \\
C 5: d \geq \delta, & C 6: \rho \leq 1, & C 7: \rho \geq 0 .
\end{array}
$$

In (10), $\zeta_{P}$ is the minimum average harvested power required at $\mathcal{D}$ to have its continued operation. With normalized slot duration assumption, $\zeta_{P}$ is equivalent to the energy requirement at $\mathcal{D}$. In $C 4$ and $C 5, \delta=\frac{2 f L^{2}}{c}$ is the minimum separation required between $\mathcal{S}$ and $\mathcal{R}$, or $\mathcal{R}$ and $\mathcal{D}$, for the antennas to be in far-field (Fraunhofer) region [27], where $L$ is the largest dimension of the antenna structure, $c$ is the speed of light, and $f$ is the frequency of the transmitted signal.

1) Equivalence of exact and asymptotic $p_{\text {out }_{1}}$ minimization: Minimizing exponential approximation of exact outage probability $p_{\text {out }_{1}}$ in (7) is equivalent to minimize its asymptotic (high SNR) version $\widehat{p_{\text {out }}}$, obtained using $e^{-x} \approx 1-x, \forall x \ll 1$,

$$
\widehat{p_{\text {out }}}=\alpha_{1}\left(\frac{d^{l}}{a_{s} P_{s}}\right)^{\beta_{1}}+\alpha_{2}\left(\frac{(D-d)^{l}}{\rho a_{r}\left(P_{T}-P_{s}\right)}\right)^{\beta_{2}} \text {. }
$$

Above observation holds because $p_{\text {out }_{1}}=1-e^{-\widehat{p_{\text {out }}}}$ is a strictly increasing function of $\widehat{p_{\text {out }}}$. As a result, the minimization problem with $p_{\text {out }}$ as objective function is equivalent [36] to the one with $\widehat{p_{\text {out }}}$ as objective function, and both problems share the same set of optimal points $\left(P_{s}^{*}, d^{*}, \rho^{*}\right)$. The optimal values, though different, are related as $p_{\text {out }_{1}}^{*}=1-e^{-{\widehat{\text { out }_{1}}}^{*}}$.

\section{Optimal Power Allocation for Fixed RP AND PS}

\section{A. Optimal PA with no direct $\mathcal{S}$-to-D link available}

Here we use the equivalence of exact and asymptotic $p_{\text {out }}$ minimization for SWIPT without $\mathcal{S}$-to- $\mathcal{D}$ direct link (see
Section III-B1) to obtain analytical expression for optimal PA. For a given $\rho$ and RP $d$ between $\mathcal{S}$ and $\mathcal{D}$, the problem of optimal PA for $\mathcal{S}$ and $\mathcal{R}$ that minimizes $\widehat{p_{\text {out }}}$ (or equivalently $p_{\text {out }_{1}}$ ), is obtained from ( $\left.\mathrm{J} 0\right)$ with $\widehat{p_{\text {out }}}$ as objective function, $P_{s}$ as optimization variable, and $C 1-C 3$ as constraints. Since $\mathcal{R}$ is placed on LOS path between $\mathcal{S}$ and $\mathcal{D}, \epsilon=1$.

Associating the Lagrange multiplier $\lambda$ with $C 1$ and keeping the boundary constraints $C 2$ and $C 3\left(0 \leq P_{s} \leq P_{T}\right)$ implicit, the Lagrangian function of (PA1) is formulated as:

$$
\begin{array}{r}
\mathcal{L}_{1}\left(P_{s}, d, \rho, \lambda\right)=\alpha_{1}\left(\frac{d^{l}}{a_{s} P_{s}}\right)^{\beta_{1}}+\alpha_{2}\left(\frac{\left(\frac{D}{\epsilon}-d\right)^{l}}{\rho a_{r}\left(P_{T}-P_{s}\right)}\right)^{\beta_{2}} \\
+\lambda\left(\zeta_{P}-\frac{\eta a_{r}(1-\rho)\left(P_{T}-P_{s}\right)}{\left.\left(\frac{D}{\epsilon}-d\right)^{l}\right)} \cdot(12)\right. \\
\text { As } \frac{\partial^{2} \widehat{p_{\text {out }}}}{\partial P_{s}^{2}}=\frac{\alpha_{1} \beta_{1}\left(\beta_{1}+1\right)\left(\frac{d^{l}}{a_{s} P_{s}}\right)^{\beta_{1}}}{P_{s}^{2}}+\frac{\alpha_{2} \beta_{2}\left(\beta_{2}+1\right)\left(\frac{\left(\frac{D}{\epsilon}-d\right)^{l}}{a_{r} \rho\left(P_{T}-P_{s}\right)}\right)^{\beta_{2}}}{\left(P_{T}-P_{s}\right)^{2}}
\end{array}
$$
$>0, \forall P_{s} \in\left[0, P_{T}\right]$ (and $\left.0<d<\frac{D}{\epsilon}\right), \widehat{p_{\text {out }}}$ is a strictly convex function of $P_{s}$ in the feasible region defined by $C 1-C 3$. Since the constraints $C 1-C 3$ are affine functions of $P_{s}$, the global optimal solution for (PA1), denoted as $P_{s}^{*}$, is obtained using the Karush-Kuhn-Tucker (KKT) conditions [37] given by: $C 1-C 3, \lambda \geq 0$,

$$
\begin{aligned}
\frac{\partial \mathcal{L}_{1}}{\partial P_{s}}= & \frac{\alpha_{2} \beta_{2}\left(\frac{D}{\epsilon}-d\right)^{\beta_{2} l}}{\left(\rho a_{r}\right)^{\beta_{2}}\left(P_{T}-P_{s}\right)^{\beta_{2}+1}}-\frac{\alpha_{1} \beta_{1} d^{\beta_{1} l}}{a_{s}^{\beta_{1}} P_{s}^{\beta_{1}+1}}+\lambda \frac{\eta a_{r}(1-\rho)}{\left(\frac{D}{\epsilon}-d\right)^{l}}=0 \\
& \text { and } \lambda\left(\zeta_{P}-\frac{\eta a_{r}(1-\rho)\left(P_{T}-P_{s}\right)}{\left(\frac{D}{\epsilon}-d\right)^{l}}\right)=0
\end{aligned}
$$

If $P_{s}^{*}=P_{T}, C 1$ cannot be satisfied $\forall \zeta_{P}>0$. Thus, $P_{s}^{*}<P_{T}$. If $\lambda^{*} \neq 0$, then

$$
P_{s}^{*}=P_{s}^{t h} \triangleq P_{T}-\frac{\zeta_{P}\left(\frac{D}{\epsilon}-d\right)^{l}}{\eta a_{r}(1-\rho)}
$$

so that (13b) is satisfied. Using (13a), $\lambda^{*}=\lambda_{P_{s}}^{t h}$ for $P_{s}^{*}=P_{s}^{t h}$ is given by (15).

Here, $P_{s}^{t h}$ is the maximum threshold power that can be allocated to $\mathcal{S}$ so that PA to $\mathcal{R}, P_{r}^{*}=P_{T}-P_{s}^{t h}$ satisfies $C 1$. As $P_{s}^{\text {th }}$ is a decreasing function of $\zeta_{P}, P A$ to $\mathcal{S}$ decreases with increasing $\zeta_{P}$ and more power is allocated to $\mathcal{R}$ to meet $C 1$ (harvested power constraint), which leads to increasing $p_{\text {out }}$ due to weakening of $\mathcal{S}$-to- $\mathcal{R}$ link. However, if $P_{s}^{t h}<0$, then (PA1) is infeasible, as $C 1$ is never satisfied. Mathematically, $P_{s}^{*}=0$ is a feasible solution, though it gives $p_{o u t_{1}}=1$. 


$$
\lambda_{P_{s}}^{t h}=\frac{\alpha_{1} \beta_{1} \rho^{\beta_{2}} \zeta_{P}^{\beta_{2}+1}\left[a_{r} \eta(1-\rho) d^{l}\right]^{\beta_{1}}\left(\frac{D}{\epsilon}-d\right)^{l}-\alpha_{2} \beta_{2} a_{s}^{\beta_{1}}\left[\eta a_{r} P_{T}(1-\rho)-\zeta_{P}\left(\frac{D}{\epsilon}-d\right)^{l}\right]^{\beta_{1}+1}[\eta(1-\rho)]^{\beta_{2}}}{a_{s}^{\beta_{1}} \rho^{\beta_{2}} \zeta_{P}^{\beta_{1}+1}\left[\left(\eta a_{r} P_{T}(1-\rho)-\zeta_{P}\left(\frac{D}{\epsilon}-d\right)^{l}\right)\right]^{\beta+1}} .
$$

If $P_{s}^{*}<P_{s}^{t h}$, then $\lambda^{*}=0$ to satisfy (13b), which on substitution in (13a) gives:

$$
\frac{\left(P_{T}-P_{s}\right)^{\beta_{2}+1}}{P_{s}^{\beta_{1}+1}}=\frac{\alpha_{2} \beta_{2}}{\alpha_{1} \beta_{1}}\left(\frac{a_{s}}{d^{l}}\right)^{\beta_{1}}\left(\frac{(D-d)^{l}}{\rho a_{r}}\right)^{\beta_{2}} .
$$

$P_{s}^{*}$ for $\lambda^{*}=0$, denoted by $P_{s_{1}}^{0}$, can be obtained by using the standard root-finding algorithms to find the efficient numerical solution of (16). However, for the same Rice factor, i.e., $K \triangleq K_{1}=K_{2}$, which implies $\alpha_{1}=\alpha_{2}$ and $\beta_{1}=\beta_{2}$, analytical closed form solution of (16) is given by: $P_{s}^{*}=$ $P_{s_{1}}^{0} \triangleq \frac{P_{T}\left(a_{r} \rho\left(\frac{d}{D-d}\right)^{l}\right)^{\frac{\beta_{1}}{\beta_{1}+1}}}{a_{s}^{\frac{\beta_{1}}{1}+1}+\left(a_{r} \rho\left(\frac{d}{D-d}\right)^{l}\right)^{\frac{\beta_{1}}{\beta_{1}+1}}}$. From the expression of $P_{s_{1}}^{0}$ it is clear that optimal PA is such that higher power is allocated to $\mathcal{S}$, if $\mathcal{R}$ is closer to $\mathcal{D}$. It may be noted that with $P_{s_{1}}^{0} \leq P_{s}^{t h}$, we have the special case where the expression for $P_{s_{1}}^{0}$, which is independent of $\zeta_{P}$, is similar to the ones obtained in [2]-[4]. This is because, the condition $P_{s_{1}}^{0} \leq P_{s}^{t h}$ arises when $\zeta_{P}$ is very low and the harvested power constraint $C 1$ is implicitly satisfied, thereby reducing the PA optimization solely to make information transfer efficient, i.e., only to minimize $p_{\text {out }}$. However, if $\zeta_{P}$ is increased, $P_{s}^{t h}$ decreases, and once it drops below $P_{s_{1}}^{0}$, the role of harvested power constraint becomes significant which influences the minimum $p_{\text {out }_{1}}$. It follows that there exists a tradeoff between minimized $p_{\text {out }}$ and the lower bound $\zeta_{P}$ on required harvested power at $\mathcal{D}$ for $P_{s_{1}}^{0}>P_{s}^{t h}$. Hence, the optimal solution of (PA1) is given by:

$$
\left(P_{s}^{*}, \lambda^{*}\right)= \begin{cases}\left(P_{s_{1}}^{0}, 0\right), & P_{s_{1}}^{0} \leq P_{s}^{t h} \\ \left(P_{s}^{t h}, \lambda_{P_{s}}^{t h}\right), & 0 \leq P_{s}^{t h}<P_{s_{1}}^{0} \\ \text { Infeasible, } & P_{s}^{t h}<0\end{cases}
$$

For $P_{s}^{t h}<P_{s_{1}}^{0}, P_{T}<\frac{\zeta_{P}(D-d)^{l}}{\eta a_{r}(1-\rho)}\left[1+\left(\frac{a_{r} \rho\left(\frac{d}{D-d}\right)^{l}}{a_{s}}\right)^{\frac{\beta_{1}}{\beta_{1}+1}}\right]$, which after some simplification gives:

$$
\zeta_{P}\left[a_{r} \rho d^{l}(D-d)^{l}\right]^{\frac{\beta_{1}}{\beta_{1}+1}}>a_{s}^{\frac{\beta_{1}}{\beta_{1}+1}}\left[\eta a_{r} P_{T}(1-\rho)-\zeta_{P}(D-d)^{l}\right] \text {. (18) }
$$

From (15) and (18), $\lambda_{P_{s}}^{t h}>0 \forall P_{s}^{t h}$, subject to $0 \leq P_{s}^{t h}<P_{s_{1}}^{0}$.

\section{B. Optimal PA with direct $\mathcal{S}$-to-D link available}

For a fixed RP and $\rho$, the problem of optimal PA at $\mathcal{S}$ and $\mathcal{R}$ with direct $\mathcal{S}$-to- $\mathcal{D}$ link available, denoted by (PA2) is similar to (PA1), but with $p_{\text {out }}$ being the objective function to be minimized. From (9), $p_{\text {out }_{2}}$ is a nonconvex function of $P_{s}$. So, we first define pseudoconvex function [37] and then claim that $p_{\text {out }}$ is a pseudoconvex function of $P_{s}$ satisfying $C 1-C 3$.

Definition 1: A differentiable function $f: \mathbb{R}^{n} \rightarrow \mathbb{R}$, defined on a nonempty open convex set $\Omega$, is called pseudoconvex if $\forall$ $x, y \in \Omega$ with $x \neq y, \nabla f(x)^{\top}(y-x) \geq 0 \Longrightarrow f(y) \geq f(x)$. A pesudoconvex function $f$ has a similar property as in convex functions, which states that, if $\exists$ a critical point, i.e., $\nabla f(\bar{x})=$ 0 , then $\bar{x}$ is a global minimum [36].
Lemma 1: $p_{\text {out }}$ is a pseudoconvex function of $P_{s} \in$ $\left.\left\{P_{s} \mid\left(P_{\text {con }}\left(P_{s}, d, \rho\right) \leq P_{T}\right) \wedge\left(0 \leq P_{s} \leq P_{T}\right)\right\}\right\}$.

Proof: See Appendix A-A.

To find the global optimal PA $\left(P_{s}^{*}, P_{r}^{*}=P_{T}-P_{s}^{*}\right)$ for a fixed RP and $\rho$ problem (PA2), while accounting the harvested power constraint $(C 1)$ and the total power constraints $(C 2$ and $C 3$ ), we use the convexity of $C 1-C 3$, along with the proposed Lemma 1 and the following lemma.

Lemma 2 ([36, Theorem 4.3.8]): Consider a constraint minimization problem $(C M P)$ with an objective function to be minimized over a feasible region $S$ being pseudoconvex at $\bar{x} \in S$, constraint functions are differentiable and quasiconvex at $\bar{x}$, and the KKT conditions hold at $\bar{x}$. Then $\bar{x}$ is a global optimal solution to $C M P$.

Associating the Lagrange multiplier $\mu$ with the harvested power constraint $C 1$ and keeping the boundary constraints $C 2-C 3$ implicit, Lagrangian function of (PA2) is given by:

$\mathcal{L}_{2}\left(P_{s}, d, \rho, \mu\right)=p_{\text {out }_{2}}+\mu\left(\zeta_{P}-\frac{\eta a_{r}(1-\rho)\left(P_{T}-P_{s}\right)}{\left(\frac{D}{\epsilon}-d\right)^{l}}\right)$

Following Lemma 2 and (19), KKT conditions (stationarity and complimentary slackness only, as the primal and dual feasibility are given by $C 1-C 3$ and $\mu \geq 0$ ) for (PA2) are:

$$
\begin{gathered}
\frac{\partial \mathcal{L}_{2}}{\partial P_{s}}=\frac{\partial p_{\text {out }_{2}}}{\partial P_{s}}+\mu\left(\frac{\eta a_{r}(1-\rho)}{\left(\frac{D}{\epsilon}-d\right)^{l}}\right)=0 \\
\mu\left(\zeta_{P}-\frac{\eta a_{r}(1-\rho)\left(P_{T}-P_{s}\right)}{\left(\frac{D}{\epsilon}-d\right)^{l}}\right)=0 .
\end{gathered}
$$

With $P_{s}^{*}=P_{T}, C 1$ cannot be satisfied $\forall \zeta_{P}>0$. Thus, $P_{s}^{*}<P_{T}$. If $\mu^{*} \neq 0$, then $P_{s}^{*}=P_{s}^{t h}$, as defined in (14) so that (21) is satisfied. $\mu^{*}=\mu_{P_{s}}^{t h}>0$ for $P_{s}^{*}=P_{s}^{t h}$ can be obtained using the value of derivative of (9) with respect to $P_{s}$ at $P_{s}^{t h}$ (i.e., $\left.\nabla_{P_{s}} p_{\text {out }}\left(P_{s}^{t h}\right)\right)$ and (20) as:

$$
\mu_{P_{s}}^{t h}=-\frac{\left[\nabla_{P_{s}} p_{\text {out }}\left(P_{s}^{t h}\right)\right]\left(\frac{D}{\epsilon}-d\right)^{l}}{\eta a_{r}(1-\rho)} .
$$

Similar to (PA1), if $P_{s}^{t h}<0$, then (PA2) is infeasible, as $C 1$ is never satisfied. $P_{s}^{*}=0$ is a feasible solution, though it gives $p_{\text {out }_{2}}=1$. If $\mu^{*}=0$, then $P_{s}^{*}<P_{s}^{\text {th }} ;(21)$ is satisfied and (20) implies finding the critical point of $p_{\text {out }}\left(P_{s}\right)$. From (9) and the discussion in Section III-A2, it can be observed that, due to the presence of highly non-linear terms in $p_{\text {out }}$, it is not possible to obtain the explicit analytic solution for (20) in $P_{s}$ with $\mu=0$. Thus, we use the Conjugate Gradient Method (CGM) with positive Polak-Ribiere (PR) beta [38] to find the global optimal solution $P_{s}^{*}$ for (PA2) by numerically solving $\frac{\partial p_{\text {out }}}{\partial P_{s}}=0$, if the critical point exists. Let us denote the global optimal PA $P_{s}^{*}$ returned by the CGM algorithm by $P_{s_{2}}^{0}$. We also use Golden-section (GS) based linear search [39] technique to restrict the search in CGM within the upper and 
lower bounds $\left(0 \leq P_{s} \leq P_{T}\right)$ such that feasibility constraints are met. Note that, this iterative algorithm provides very good convergence due to the pseudoconvexity of the problem.

$P_{s_{2}}^{0}$ is independent of $\zeta_{P}$ and, since for $P_{s_{2}}^{0}<P_{s}^{t h}$, $C 1$ is not active, it implies that $P_{s}^{*}=P_{s_{2}}^{0}$ provides the minimum $p_{\text {out }_{2}}$ for a predetermined RP and $\rho$. Similar to (PA1), a tradeoff between minimized $p_{\text {out }_{2}}$ and $\zeta_{P}$ exists for $P_{s_{2}}^{0}>P_{s}^{t h}$. The optimal solution is given by:

$$
\left(P_{s}^{*}, \mu^{*}\right)= \begin{cases}\left(P_{s_{2}}^{0}, 0\right), & P_{s_{2}}^{0} \leq P_{s}^{t h} \\ \left(P_{s}^{t h}, \mu_{P_{s}}^{t h}\right), & 0 \leq P_{s}^{t h}<P_{s_{2}}^{0} \\ \text { Infeasible, } & P_{s}^{t h}<0 .\end{cases}
$$

\section{Optimal Relay Placement for FiXed PA AND PS}

\section{A. Optimal RP with no direct $\mathcal{S}$-to-D link available}

For a predetermined PA $\left(P_{s}, P_{r}\right)$ and $\rho$, we now obtain optimal RP, i.e., distance $d^{*}$ between $\mathcal{S}$ and $\mathcal{R}$, or $\left(D-d^{*}\right)$ between $\mathcal{R}$ and $\mathcal{D}$, with $\mathcal{R}$ placed on the direct $\mathcal{S}$-to- $\mathcal{D}$ path (Fig. 1(a)). The optimal RP $\left(d^{*}, D-d^{*}\right)$ problem, denoted as (RP1), is obtained from (10), with $\widehat{p_{\text {out }}}$ as objective function to be minimized over the variable $d$ subject to $C 1, C 4-C 5$.

As $\frac{\partial^{2} \widehat{p_{\text {out }}}}{\partial d^{2}}=\frac{\alpha_{1} \beta_{1} l\left(\beta_{1} l-1\right) d^{\beta_{1} l-2}}{\left(a_{s} P_{s}\right)^{\beta_{1}}}+\frac{\alpha_{2} \beta_{2} l\left(\beta_{2} l-1\right)\left(\frac{D}{\epsilon}-d\right)^{\beta_{2} l-2}}{\left(a_{r} \rho\left(P_{T}-P_{s}\right)\right)^{\beta_{2}}}>$ $0, \forall d \in\left[\delta, \frac{D}{\epsilon}-\delta\right]\left(\right.$ and $\left.(l>1) \wedge\left(P_{s} \in\left[0, P_{T}\right]\right)\right), \widehat{p_{\text {out }}}$ is a strictly convex function of $d$ in the feasible region defined by $C 1, C 4-C 5$. Since $C 1, C 4-C 5$ are convex functions of $d$, the global solution for (RP1), $d^{*}$, can be obtained using the KKT conditions given by (24), (13b), C1,C4-C5, and $\lambda \geq 0$.

$$
\begin{aligned}
\frac{\partial \mathcal{L}_{1}}{\partial d}= & \frac{\alpha_{1} \beta_{1} l d^{\beta_{1} l-1}}{\left(a_{s} P_{s}\right)^{\beta_{1}}}-\frac{\alpha_{2} \beta_{2} l\left(\frac{D}{\epsilon}-d\right)^{\beta_{2} l-1}}{\left[\rho a_{r}\left(P_{T}-P_{s}\right)\right]^{\beta_{2}}} \\
& +\lambda\left(-\frac{\eta a_{r} l(1-\rho)\left(P_{T}-P_{s}\right)}{\left(\frac{D}{\epsilon}-d\right)^{l+1}}\right)=0 .
\end{aligned}
$$

If $\lambda^{*} \neq 0, d^{*}=d^{\text {th }}$ that satisfies (13b) is defined as follows:

$$
d^{*}=d^{t h} \triangleq \frac{D}{\epsilon}-\left(\frac{\eta a_{r}(1-\rho)\left(P_{T}-P_{s}\right)}{\zeta_{P}}\right)^{1 / l} .
$$

Using (24), for $d^{*}=d^{t h}, \lambda^{*}=\lambda_{d}^{t h}$ is given by (26). Note that, $d^{\text {th }}$ is the minimum threshold distance of $\mathcal{R}$ from $\mathcal{S}$, such that the received power $P_{r}$ at $\mathcal{D}$ satisfies $C 1 . d^{\text {th }}$ is an increasing function of $\zeta_{P}$ and, if $d^{\text {th }}>\frac{D}{\epsilon}-\delta$, then (RP2) is infeasible. If $d^{*}>d^{t h}$, then $\lambda^{*}=0$, which, by using (24) gives,

$$
\frac{d^{\beta_{1} l-1}}{(D-d)^{\beta_{2} l+1}}=\frac{\alpha_{2} \beta_{2}\left(a_{s} P_{s}\right)^{\beta_{1}}}{\alpha_{1} \beta_{1}\left(\rho a_{r}\left(P_{T}-P_{s}\right)\right)^{\beta_{2}}} .
$$

Although closed-form analytical solution cannot be obtained for (27), an efficient numerical solution, denoted by $d_{1}^{0}$, can be obtained using easily available standard rootfinding algorithms. If we again consider same Rice factor for all the links, i.e., $\alpha_{1}=\alpha_{2}$ and $\beta_{1}=\beta_{2}$, analytical closed form solution of (27) is given by: $d^{*}=d_{1}^{0} \triangleq$ $\max \left[\delta, \min \left\{\frac{D\left(a_{s} P_{s}\right)^{\frac{\beta_{1}}{\beta_{1} l-1}}}{\left[a_{r} \rho\left(P_{T}-P_{s}\right)\right]^{\frac{\beta_{1}}{\beta_{1} l-1}}+\left(a_{s} P_{s}\right)^{\frac{\beta_{1}}{\beta_{1} l-1}}}, D-\delta\right\}\right], \quad$ so that $d_{1}^{0}$ does not violate upper and lower bounds on $d$. Optimal solution of (RP1) is given by:

$$
\left(d^{*}, \lambda^{*}\right)= \begin{cases}\left(d_{1}^{0}, 0\right), & d_{1}^{0} \geq d^{t h} \\ \left(d^{t h}, \lambda_{d}^{t h}\right), & d_{1}^{0}<d^{t h} \leq D-\delta \\ \text { Infeasible, }, & d^{t h}>D-\delta .\end{cases}
$$

(28) gives the feasible region for (RP1) if $P_{s}<P_{T}$ (or $\left.P_{r}>0\right)$. If $d^{t h}>d_{1}^{0}$, then $D>\left(\frac{\eta a_{r}(1-\rho)\left(P_{T}-P_{s}\right)}{\zeta_{P}}\right)^{\frac{1^{T}}{l}}[1+$ $\left.\left(\frac{a_{s} P_{s}}{a_{r} \rho\left(P_{T}-P_{s}\right)}\right)^{\frac{\beta_{1}}{\beta_{1} l-1}}\right]$, which after some rearrangement gives:

$$
d^{t h}\left[a_{r} \rho\left(P_{T}-P_{s}\right)\right]^{\frac{\beta_{1}}{\beta_{1} l-1}}>\left(D-d^{t h}\right)\left(a_{s} P_{s}\right)^{\frac{\beta_{1}}{\beta_{1} l-1}} .
$$

From (26) and (29), $\lambda_{d}^{t h}>0 \forall d^{t h}$, with $d_{1}^{0}<d^{t h} \leq D-\delta$. Similar to as noted in Section IV-A, with $d_{1}^{0} \geq d^{t h}$, we have a special case where the expression for $d_{1}^{0}$ is similar to the ones obtained in [2]-[4]. This is because, the condition $d_{1}^{0} \geq d^{t h}$ arises when $\zeta_{P}$ is very low and $C 1$ is implicitly met, so optimal $\mathrm{RP}$ is carried out solely to minimize $p_{\text {out }}$. Also, optimal RP in this case, $d^{*}=d_{1}^{0}$, is such that for higher PA to $\mathcal{S}, \mathcal{R}$ is placed closer to $\mathcal{D}$. But, as $\zeta_{P}$ is increased depending on the energy requirements at $\mathcal{D}, d^{\text {th }}$ increases. If $d^{t h}>d_{1}^{0}$, then optimal RP $d^{*}=d^{t h}$, is dependent on $\zeta_{P}$, and thus there exists a tradeoff between the minimized $p_{\text {out }}$ and $\zeta_{P}$.

\section{B. Optimal $R P$ with $\mathcal{S}$-to- $\mathcal{D}$ direct link available}

For a predetermined PA $\left(P_{s}, P_{r}\right)$ and $\rho$, optimal RP problem (RP2) of finding the optimal distance $d^{*}$ between $\mathcal{S}$ and $\mathcal{R}$, or $\left(\frac{D}{\epsilon}-d^{*}\right)$ between $\mathcal{R}$ and $\mathcal{D}$, with $\mathcal{R}$ placed on the elliptical path with $\mathcal{S}$ and $\mathcal{D}$ as the foci (see Fig. 1(b)) that minimizes $p_{\text {out }_{2}}$, has same optimization variable and constraints as (RP1), except the objective function to be minimized being $p_{\text {out }}$.

The constraint function defined in $C 1$ is convex in $d$, and $C 4$ and $C 5$ are affine functions of $d$. In the following lemma, we claim that $p_{\text {out }_{2}}$ is pseudoconvex in $d$ in the feasible RP region $\mathcal{F}_{d}=\left\{d \mid\left(P_{\text {con }}\left(P_{s}, d, \rho\right) \leq 0\right) \wedge\left(\delta \leq d \leq \frac{D}{\epsilon}-\delta\right)\right\}$ as defined by the constraints $C 1, C 4$, and $C 5$.

Lemma 3: Outage probability $p_{\text {out }_{2}}$ is a pseudoconvex function of $\mathcal{S}$-to- $\mathcal{R}$ distance $d \in \mathcal{F}_{d}$.

Proof: See Appendix B-A.

The pseudoconvexity of $p_{\text {out }_{2}}$ in $d$ is due to the log-concavity of complimentary CDFs of $\gamma_{1}$ and $\Upsilon_{02}$, i.e., $\mathcal{C}_{\gamma_{1}}$ and $\mathcal{C}_{\Upsilon_{02}}$, respectively, in $\mathcal{S}$-to- $\mathcal{R}$ distance $d$ (see Appendix $B$ for details).

The KKT conditions for (RP2) are given by (30) and (21), along with $C 1, C 4-C 5$, and $\mu \geq 0$.

$$
\frac{\partial \mathcal{L}_{2}}{\partial d}=\frac{\partial p_{\text {out }_{2}}}{\partial d}+\mu\left(-\frac{\eta a_{r} l(1-\rho)\left(P_{T}-P_{s}\right)}{\left(\frac{D}{\epsilon}-d\right)^{l+1}}\right)=0 .
$$

If $\mu^{*}>0, d^{*}=d^{t h}$ as defined in (25) with $\epsilon<1$, so that $C 1$ and (13b) are satisfied. The value of $\mu^{*}=\mu_{d}^{t h}$ at $d^{*}=d^{t h}$ is obtained using $\nabla_{d} p_{\text {out }_{2}}\left(d^{\text {th }}\right)$ and (30) as:

$$
\mu_{d}^{t h}=\frac{\left[\nabla_{d} p_{\text {out }_{2}}\left(d^{\text {th }}\right)\right]\left(\frac{D}{\epsilon}-d\right)^{l+1}}{\eta a_{r} l(1-\rho)\left(P_{T}-P_{s}\right)} .
$$

If $\mu^{*}=0$, then $d^{*}>d^{\text {th }}$; (21) is satisfied and (30) implies finding the critical point of $p_{\text {out }_{2}}$, i.e., $\frac{\partial p_{\text {out }_{2}}}{\partial d}=0$. Observe from (9) that similar to $p_{\text {out }}, \frac{\partial p_{\text {out }_{2}}}{\partial d}$ contains highly non-linear terms. Therefore, it is not possible to obtain the explicit analytic solution of (30) for $d^{*}$ with $\mu=0$. Again, 


$$
\lambda_{d}^{t h}=\frac{\alpha_{1} \beta_{1}\left(D-d^{t h}\right)\left(d^{t h}\right)^{\beta_{1} l}\left(a_{r} \rho\left(P_{T}-P_{s}\right)\right)^{\beta_{2}}-\alpha_{2} \beta_{2}\left(a_{s} P_{s}\right)^{\beta_{1}} d^{t h}\left(D-d^{t h}\right)^{\beta_{2} l}}{\eta(1-\rho)\left(a_{s} P_{s}\right)^{\beta_{1}}\left(a_{r}\left(P_{T}-P_{s}\right)\right)^{\beta_{2}+1} d^{t h}\left[\left(D-d^{t h}\right)^{l}\right]^{-1}} .
$$

we use CGM with positive PR beta and GS based linear search techniques to find $d^{*}$ for (RP2) by indirectly solving $\frac{\partial p_{\text {out }}}{\partial d}=0$ (if the critical point exists), while restricting the search within the upper and lower bounds $\left(\delta \leq d \leq \frac{D}{\epsilon}-\delta\right)$. We denote the global optimal PA $d^{*}$ obtained from CGM algorithm by $d_{2}^{0}$. So, optimal solution of (RP2) is given by:

$$
\left(d^{*}, \mu^{*}\right)= \begin{cases}\left(d_{2}^{0}, 0\right), & d_{2}^{0} \geq d^{t h} \\ \left(d^{t h}, \mu_{d}^{t h}\right), & d_{2}^{0}<d^{t h} \leq \frac{D}{\epsilon}-\delta \\ \text { Infeasible, }, & d^{t h}>\frac{D}{\epsilon}-\delta .\end{cases}
$$

Thus, (32) gives the feasible region for (RP2) if $P_{s}<P_{T}$ (i.e., some power is allocated to $\mathcal{R}$ ). Also, $\mu_{d}^{t h}>0 \forall d^{t h}$, with $d_{2}^{0}<d^{t h} \leq \frac{D}{\epsilon}-\delta . d_{2}^{0}$, independent of $\zeta_{P}$, corresponds to the case when $C 1$ is not active, thus providing the minimum outage probability for a predetermined PA and $\rho$. Similar to as noted in Section V-A, with increased energy requirement $\zeta_{P}$ at $\mathcal{D}, d^{t h}$ increases and, if $d_{2}^{0}<d^{t h} \leq \frac{D}{\epsilon}-\delta$, then there exists a tradeoff between minimized $p_{\text {out }_{2}}$ and $\zeta_{P}$.

\section{Optimal PS RATio FOR FiXed PA AND RP}

In this section we derive optimal PS for a predetermined PA and RP. The PS optimization problem (PSO) is formulated using (10) with $\rho$ as the optimization variable and $C 1, C 6-C 7$ as constraints. Due to the monotonicity of outage probability in $\rho$, we consider the same optimization problem (PS0) for both with and without direct $\mathcal{S}$-to- $\mathcal{D}$ link cases. As $\rho$ is the ratio of total power received at $\mathcal{D}$, which is utilized for information decoding, higher $\rho$ gives lesser $p_{\text {out }_{i}} \forall i=1,2$. Next we discuss convexity of (PSO) and then obtain optimal $\rho^{*}$.

A. Convexity of $\widehat{\text { out }_{1}}$ in $\rho$

$$
\text { As } \frac{\partial^{2} \widehat{\text { out }_{1}}}{\partial \rho^{2}}=\frac{\alpha_{2} \beta_{2}\left(\beta_{2}+1\right)}{\rho^{2}}\left(\frac{(D-d)^{l}}{\rho a_{r}\left(P_{T}-P_{s}\right)}\right)^{\beta_{2}}>0, \forall \rho \in[0,1]
$$

(and $0<P_{s}<P_{T}, \delta<d<D-\delta$ ), $\widehat{p_{\text {out }}}$ is a strictly convex function of $\rho$ in the feasible region defined by $C 1, C 6-C 7$. Since the constraints $C 1, C 6-C 7$ are linear functions of $\rho$, and the gradient of $\widehat{p_{\text {out }}}$ does not vanish in the feasible region, the global solution for (PS0), $\rho^{*}$, is given by the corner point obtained by solving $C 1$ at strict equality, i.e., $\rho^{*}=\rho^{\text {th }} \triangleq 1-$ $\frac{\zeta_{P}\left(\frac{D}{\epsilon}-d\right)^{l}}{\eta a_{r}\left(P_{T}-P_{s}\right)}$. Here $\rho^{t h}$ is the maximum portion of the average received power at $\mathcal{D}$ that can be allocated for data decoding while satisfying $C 1$. With $\mathcal{L}_{1}$ as the Lagrangian function for (PS0), the Lagrange multiplier $\lambda_{\rho}^{t h}$ in this case is:

$$
\lambda_{\rho}^{t h}=\frac{\alpha_{2} \beta_{2}}{\eta}\left(\frac{(D-d)^{l}}{a_{r} \rho^{t h}\left(P_{T}-P_{s}\right)}\right)^{\beta_{2}+1}>0 \forall\left\{(d \leq D) \wedge\left(P_{s} \leq P_{T}\right)\right\} .
$$

\section{B. Pseudoconvexity of $p_{\text {out }}$ in $\rho$}

Lemma 4: Outage probability $p_{\text {out }_{2}}$ is a pseudoconvex function of $\rho \in \mathcal{F}_{\rho}=\{\rho \mid 0 \leq \rho \leq 1\}$.

Proof: See Appendix C.

So, using Lemma 2 and Lemma 4, the KKT point of (PSO) provides the global optimal solution. However, like in Section VI-A, here also the KKT point is obtained by solving $C 1$ for $\rho$ at strict equality, which gives $\rho^{*}=\rho^{t h}$. The Lagrange multiplier $\mu_{\rho}^{t h}$, obtained by solving $\frac{\partial \mathcal{L}_{2}}{\partial \rho}=0$, is:

$$
\mu_{\rho}^{\text {th }}=-\frac{\left[\nabla_{\rho} p_{\text {out }_{2}}\left(\rho^{\text {th }}\right)\right]\left(\frac{D}{\epsilon}-d\right)^{l}}{\eta a_{r}\left(P_{T}-P_{s}\right)}>0 \text {, because } \nabla_{\rho} p_{\text {out }_{2}}\left(\rho^{\text {th }}\right)<0
$$

It may be noted that PS optimization has least complexity in terms of implementation, among the three proposed semiadaptive schemes. The optimal solution of (PSO) is given by:

$\left(\rho^{*}, \lambda^{*}\right)= \begin{cases}\left(\rho^{t h}, \lambda_{\rho}^{t h}\right), & \rho^{t h} \geq 0 \text { with no direct } \mathcal{S} \text {-to- } \mathcal{D} \text { link, } \\ \left(\rho^{t h}, \mu_{\rho}^{t h}\right), & \rho^{t h} \geq 0 \text { with direct link availability, }(35) \\ \text { Infeasible, } & \rho^{t h}<0 .\end{cases}$

\section{JOINT OPTIMIZATION OF PA, RP, AND PS}

Here we derive the joint-optimal solutions with and without $\mathcal{S}$-to- $\mathcal{D}$ direct link.

\section{A. Joint optimization with no direct $\mathcal{S}$-to-D link available}

As noted in Section VI, outage probability is a strictly decreasing function of $\rho$, which implies that $C 1$ in joint optimization problem should be satisfied with strict equality. This reduces three-variable minimization problem (J0) for $p_{\text {out }_{1}}$ in (7) into an equivalent two-variable problem (J1).

$$
\begin{aligned}
&(\mathbf{J 1}): \underset{P_{s}, d}{\operatorname{minimize}} \quad \widehat{p_{\text {out }_{1 J}} \triangleq \alpha_{1}\left(\frac{d^{l}}{a_{s} P_{s}}\right)^{\beta_{1}}+} \\
& \alpha_{2}\left(\frac{\eta(D-d)^{l}}{\eta a_{r}\left(P_{T}-P_{s}\right)-\zeta_{P}(D-d)^{l}}\right)^{\beta_{2}}
\end{aligned}
$$

subject to $C 2, C 3, C 4, C 5$, and

$$
C 8: g_{C 8} \triangleq \frac{\zeta_{P}\left(\frac{D}{\epsilon}-d\right)^{l}}{\eta a_{r}\left(P_{T}-P_{s}\right)}-1 \leq 0 .
$$

Theorem 1: Outage probability $\widehat{p_{\text {out }} \text { IJ }}$ is a convex function of source power $P_{s}$ and $\mathcal{S}$-to- $\mathcal{R}$ distance $d$ over feasible region defined by the convex constraints $C 2-C 5$ and $C 8$. So, the KKT point yields the global optimal solution of (J1).

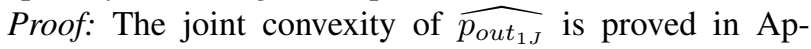
pendix D. $C 2-C 5$ are affine functions of $P_{s}$ and $d$. Whereas, $C 8$ is a convex function of $P_{s}$ and $d$ (see Appendix E). Using these results, along with Lemma 2, proves that KKT point is the global optimal solution of (J1).

Constraint $C 8$ along with $C 2-C 3$ provide upper and lower bounds on $P_{s}$, given as: $0 \leq P_{s} \leq \max \left\{0, P_{T}-\frac{\zeta_{P}(D-d)^{l}}{\eta a_{r}}\right\}$. Similarly, bounds on $d$, obtained using $C 4-C 5$ and $C 8$, are given as $\max \left\{\delta, D-\left(\frac{\eta a_{r}\left(P_{T}-P_{s}\right)}{\zeta_{P}}\right)^{\frac{1}{l}}\right\} \leq d \leq D-\delta$. Keeping these boundary constraints implicit, (J1) can be solved as an unconstrained problem, whose Lagrangian function is $\widehat{p_{\text {out }} \text { IJ }}$ itself. So, the stationarity KKT conditions for (J1) are:

$$
\begin{gathered}
\frac{\alpha_{2} \beta_{2} \eta^{\beta_{2}+1} a_{r}(D-d)^{\beta_{2} l}}{\left(\eta a_{r}\left(P_{T}-P_{s}\right)-\zeta_{P}(D-d)^{l}\right)^{\beta_{2}+1}}-\frac{\alpha_{1} \beta_{1} d^{\beta_{1} l}}{a_{s}^{\beta_{1}} P_{s}^{\beta_{1}+1}}=0, \\
\frac{\alpha_{1} \beta_{1} l d^{\beta_{1} l-1}}{\left(a_{s} P_{s}\right)^{\beta_{1}}}-\frac{\alpha_{2} \beta_{2} \eta^{\beta_{2}+1} a_{r}\left(P_{T}-P_{s}\right) l(D-d)^{\beta_{2} l-1}}{\left(\eta a_{r}\left(P_{T}-P_{s}\right)-\zeta_{P}(D-d)^{l}\right)^{\beta_{2}+1}}=0 .
\end{gathered}
$$


On solving (37a), (37b) for $P_{s}=P_{s}^{J}$ and $d=d^{J}$ with $\alpha_{1}=\alpha_{2}$, $\beta_{1}=\beta_{2}$, we obtain $d^{J} \triangleq \frac{D P_{s}^{J}}{P_{T}}$, where $P_{s}^{J}$ is obtained by finding root of (38) in interval $\left[\max \left\{0, P_{T}-\left[\left(\frac{P_{T}}{D}\right)^{l} \frac{a_{r} \eta}{\zeta_{P}}\right]^{\frac{1}{l-1}}\right\}, P_{T}\right]$.

$$
\frac{\eta^{\beta_{2}+1} D^{\left(\beta_{2}-\beta_{1}\right) l} a_{s}^{\beta_{1}} a_{r} P_{T}^{\left(\beta_{1}+1\right) l}\left(P_{T}-P_{s}^{J}\right)^{\beta_{2}(l-1)-1}}{\left(\eta a_{r} P_{T}^{l}-\zeta_{P} D^{l}\left(P_{T}-P_{s}^{J}\right)^{l-1}\right)^{\beta_{2}+1}\left(P_{s}^{J}\right)^{\beta_{1}(l-1)-1}}=\frac{\alpha_{1} \beta_{1}}{\alpha_{2} \beta_{2}} .
$$

So, $d^{*}=\max \left[\delta, \min \left\{d^{J}, D-\delta\right\}\right]$, using which $P_{s}^{*}$ is obtained as,

$$
P_{s}^{*}= \begin{cases}\frac{\eta a_{r} p_{T}-\zeta_{P}\left(D-d^{*}\right)^{l}}{\left(\frac{a_{r} a_{s}^{\beta_{1}} \eta^{\beta_{1}+1}\left(D-d^{*} \beta_{1} l\right.}{\left(d^{*}\right)^{\beta} 1^{l}}\right)^{\frac{1}{\beta_{1}+1}}+\eta a_{r}}, & {\left[\left(d^{*}=\delta\right) \vee\right.} \\ & \left.\left(d^{*}=D-\delta\right)\right] \\ P_{s}^{J}, & \delta<d^{*}<D-\delta .\end{cases}
$$

Following this, $\rho^{*}=1-\frac{\zeta_{P}\left(D-d^{*}\right)^{l}}{\eta a_{r}\left(P_{T}-P_{s}^{*}\right)}$. So, $\left(P_{s}^{*}, d^{*}, \rho^{*}\right)$ is the joint-optimal solution of $(J 1)$ if $\zeta_{P} \leq \frac{\eta a_{r} P_{T}}{\delta^{l}}$; otherwise the problem is infeasible. As $P_{s}^{*}, d^{*}$, and $\rho^{*}$ are all functions of $\zeta_{P}$, there exists a tradeoff between minimized $p_{o u t_{1}}$ and $\zeta_{P}$. Minimized $p_{\text {out }}$ obtained from the joint optimization is better (lower) than the three partially-adaptive optimization schemes (only PA, or only RP, or only PS), as shown via numerical results in Section VIII-E. Indeed, besides utilizing the optimal amount of received energy for harvesting, simultaneously $\mathcal{R}$ can be moved closer to $\mathcal{D}$ and the weaker $\mathcal{S}$-to- $\mathcal{R}$ link can be improved by allocating a higher power to $\mathcal{S}$.

\section{B. Joint optimization with $\mathcal{S}$-to- $\mathcal{D}$ direct link available}

Using the problem definitions for $(\mathrm{J} 0)$ and $(\mathrm{J} 1)$ provided in Sections III-B and VII-A, respectively, an equivalent twovariable joint optimization problem that minimizes $p_{\text {out }_{2}}$ is:

$$
\begin{aligned}
& (\mathbf{J 2}): \underset{P_{s}, d}{\operatorname{minimize}} p_{\text {out }_{2 J}} \\
& \text { subject to } C 2, C 3, C 4, C 5, C 8 \text {. }
\end{aligned}
$$

Here, $p_{\text {out }}$, obtained by substituting $\rho=1-\frac{\zeta_{P}\left(\frac{D}{\epsilon}-d\right)^{l}}{\eta a_{r}\left(P_{T}-P_{s}\right)}$ in (9), is jointly nonconvex in $P_{s}$ and $d$. Here we first define a bi-pseudoconvex function and then we use it in Theorem 2 .

Definition 2: A function $f(x, y)$ with $x \in X$ and $y \in$ $Y$, defined over a bi-convex set $B \subset X \times Y$, is called a bi-pseudoconvex if upon fixing $x=\bar{x}, f_{x}(y)=f(\bar{x}, y)$ is pseudoconvex over $Y$, and fixing $y=\bar{y}, f_{y}(x)=f(x, \bar{y})$ is pseudoconvex over $X$.

Theorem 2: Outage probability $p_{o u t_{2 J}}$ is a bi-pseudoconvex function of source power $P_{s}$ and $\mathcal{S}$-to- $\mathcal{R}$ distance $d$ over the bi-convex set $B^{\prime}$ defined by the constraints $C 2-C 5$ and $C 8$.

Proof: Outage probability $p_{o u t_{2 J}}: B^{\prime} \rightarrow[0,1]$ is a bipseudoconvex function of $P_{s}$ and $d$, because: (i) $p_{o u t_{2 J}}$ is pseudoconvex in $P_{s}$ for every fixed $d$ (see Appendix A-B), (ii) $p_{\text {out }_{2 J}}$ is a pseudoconvex function of $d$ for every fixed $P_{s}$ (see Appendix B-B), and (iii) feasible region $B^{\prime}$ defined by $C 1-C 5$ and $C 8$ is a convex set (see Section VII-A).

Remark 1: Generalizing the concept of bi-pseudoconvexity, from Lemmas 1, 3, and 4, it may be noted that $p_{\text {out }}$ is a multipseudoconvex (or tri-pseudoconvex) function of $P_{s}, d$, and $\rho$, because it is individually pseudoconvex in each of them $\left(P_{s}\right.$, $d$, and $\rho$ ), with the other two being fixed.
Using Theorem 2 and Lemma 2, the global optimal solution for ( $\mathbf{J} 2),\left(P_{s}^{*}, d^{*}\right)$, is obtained using KKT conditions. It may be noted that $C 2$ and $C 3$ cannot be satisfied at strict equality because they respectively lead to $\rho<0$ and $p_{o u t_{2 J}}=1$. Moreover, if $C 8$ is satisfied with strict equality, it will lead to $\rho=0$, which cannot meet $C 1 \forall \zeta_{P}>0$. Thus, only $d$ can be satisfied at its two extremes, i.e., $\delta$ and $\frac{D}{\epsilon}-\delta$. So, keeping the boundary constraints $C 3-C 4$ on $d$ implicit, finding the KKT point reduces to finding the critical point of $p_{\text {out } t_{2 J}}\left(P_{s}, d\right)$ if it exists, or finding the minimum $p_{\text {out }}$ J subject to boundary constraints $C 2-C 5$ and $C 8$. Minimization of $p_{o u t_{2 J}}$ over both $P_{s}$ and $d$ simultaneously is nonconvex, however minimization of $p_{\text {out }_{2 J}}$ with respect to either of them while keeping the other one fixed is pseudoconvex. In this situation, following Theorem 2 and exploiting the merits of bi-pseudoconvexity of $p_{\text {out }_{2 J}}$, we next propose an alternating optimization algorithm described in Algorithm 1 to find the joint-optimal solution.

$\overline{\text { Algorithm } 1 \text { Alternating optimization to find joint-optimal PA, }}$ RP, and PS to minimize $p_{\text {out }_{2}}$.

Input: $d_{0}$ and $\xi$

Output: $p_{\text {out }_{2 J}}^{*}, P_{s}^{*}, d^{*}, \rho^{*}$

1: Set $i \leftarrow 0, p_{\text {out }}^{(0)} \leftarrow p_{\text {out }_{2} J}\left(\frac{1}{2} \max \left\{0, P_{T}-\frac{\zeta_{P}\left(\frac{D}{\epsilon}-d_{0}\right)^{l}}{\eta a_{r}}\right\}, d_{0}\right)$
2: repeat (Main Loop)

3: $\quad$ Set $i \leftarrow i+1$

4: Apply CGM with positive PR beta and GS method to find optimal PA satisfying $C 2, C 3, C 8$, for fixed RP $d=d_{i-1}$ and fixed PS $\rho=1-\frac{\zeta_{P}\left(\frac{D}{\epsilon}-d_{i-1}\right)^{l}}{\eta a_{r}\left(P_{T}-P_{s}\right)}$ : $\left.\left[P_{s_{i}} \leftarrow \underset{0 \leq P_{s} \leq \max \left\{0, P_{T}-\frac{\zeta_{P}\left(\frac{D}{\epsilon}-d_{i-1}\right)^{l}}{\eta a_{r}}\right\}}{\operatorname{argmin}}\right\} p^{\text {out }_{2 J}\left(P_{s}, d_{i-1}\right)}\right]$

5: Apply CGM with positive PR beta and GS method to find optimal RP satisfying $C 4, C 5, C 8$, for fixed PA $P_{s}=P_{s_{i}}$ and fixed PS $\rho=1-\frac{\zeta_{P}\left(\frac{D}{\epsilon}-d\right)^{l}}{\eta a_{r}\left(P_{T}-P_{s_{i}}\right)}$ : $\left[d_{i} \leftarrow \underset{\max \left\{\delta, \frac{D}{\epsilon}-\left(\frac{\left.\eta a_{r}\left(P_{T}-P_{s_{i}}\right)\right)}{\zeta_{P}}\right)^{\frac{1}{l}}\right\} \leq d \leq \frac{D}{\epsilon}-\delta}{\operatorname{argmin}}{\operatorname{pout~}{ }_{2 J}\left(P_{s_{i}}, d\right)}\right.$ 6: $\quad$ Set $p_{\text {out }}^{(i)} \leftarrow p_{\text {out }_{2 J}}\left(P_{s_{i}}, d_{i}\right), \quad p_{\text {out }_{2 J}}^{*} \leftarrow p_{\text {out }}^{(i)}$, 7: $\quad$ Set $P_{s}^{*} \leftarrow P_{s_{i}}, \quad d^{*} \leftarrow d_{i}, \quad \rho^{*} \leftarrow 1-\frac{\zeta_{P}\left(\frac{D}{\epsilon}-d_{i}\right)^{l}}{\eta a_{r}\left(P_{T}-P_{s_{i}}\right)}$ 8: until $\left(p_{\text {out }}^{(i)}-p_{\text {out }}^{(i-1)}\right) \leq \xi$.

Algorithm 1 starts with a feasible starting point given by: $d_{0} \triangleq \frac{1}{2}\left(\delta+\max \left\{\delta, \frac{D}{\epsilon}-\left(\frac{\eta a_{r} P_{T}}{\zeta_{P}}\right)^{\frac{1}{l}}\right\}\right)$ and generates an alternating minimization sequence of PA and RP, i.e., $P_{s_{1}} \rightarrow d_{1} \rightarrow P_{s_{2}} \rightarrow d_{2} \rightarrow \cdots$. It returns the jointoptimal PA, RP, and PS $\left(P_{s}^{*}, d^{*}, \rho^{*}\right)$ along with the minimum outage probability $p_{\text {out } t_{2 J}}^{*}$. It can be observed that the sequence $p_{\text {out }}^{(i)}$ is non-increasing and converges to global minimum [40] because $p_{\text {out }_{2 J}}$ is individually a pseudoconvex function of $P_{s}$ and $d$, and is bounded from below, i.e., $p_{\text {out }_{2 J}} \geq 0$. Algorithm 1 terminates when $\left(p_{\text {out }}^{(i)}-p_{\text {out }}^{(i-1)}\right) \leq \xi$, where $\xi$ is an acceptable tolerance. If $\zeta_{P} \leq \frac{\eta a_{r} P_{T}}{\delta^{l}}$, then joint-optimal solution returned by Algorithm 1 is a feasible KKT point and, 
TABLE II: Summary of proposed joint cooperative optimization schemes for SWIPT over Rician channels.

\begin{tabular}{|c|c|c|c|c|}
\hline \multicolumn{2}{|c|}{ Optimization scheme } & \multicolumn{2}{|c|}{ Features of optimization problem } & Remarks on optimal solution(s) \\
\hline \multirow{4}{*}{ 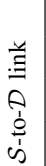 } & \multirow{3}{*}{$\begin{array}{l}\text { Optimal PA } \\
\text { (PA1) }\end{array}$} & easibility condition & & \multirow{3}{*}{$\begin{array}{l}P_{s}^{*}=P_{s_{1}}^{0} \text { implies that higher power is allocated to transmitter } \\
\text { of the weaker link, i.e., } P_{s}^{*} \geq P_{r}^{*} \text { if } \frac{a_{s}}{d^{l}} \leq \frac{a_{r} \rho}{(D-d)^{t}} \text { and vice-versa. } \\
P_{s}^{*}=P_{s}^{t h} \text { implies that sufficient power is allocated to } \mathcal{R} \text { to meet } \zeta_{P} \text {. }\end{array}$} \\
\hline & & Objective function & convex & \\
\hline & & Conve & & \\
\hline & \multirow{3}{*}{$\begin{array}{l}\text { Optimal RP } \\
\text { (RP1) }\end{array}$} & easibili & $d^{t h} \leq D-\delta$ & \multirow{3}{*}{$\begin{array}{l}d^{*}=d_{1}^{0} \text { implies that } \mathcal{R} \text { is placed closer to } \mathcal{S} \text { if } \mathcal{S} \text {-to- } \mathcal{R} \text { link is weaker } \\
\text { than the } \mathcal{R} \text {-to- } \mathcal{D} \text { link, }\left(d^{*} \leq D-d^{*} \text { if } a_{s} P_{s} \leq a_{r} \rho\left(P_{T}-P_{s}\right)\right) \\
d^{*}=d^{t h} \text { implies that } \mathcal{R} \text { is placed sufficiently close to } \mathcal{D} \text { to meet } \zeta_{P} \text {. }\end{array}$} \\
\hline \multirow{5}{*}{ 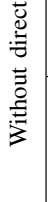 } & & Objective function & convex & \\
\hline & & Con & $C 1, C 4-C$ & \\
\hline & \multirow{3}{*}{$\begin{array}{l}\text { Joint optimization } \\
\text { of PA, RP, and PS } \\
\text { (J1) }\end{array}$} & Feasibility condition & $\zeta_{P} \leq \frac{\eta a_{r} P_{T}}{\delta^{l}}$ & \multirow{3}{*}{$\begin{array}{l}P_{s}^{*} \text { obtained using }(39), d^{*}=\max \left[\delta, \min \left\{d^{J}, D-\delta\right\}\right] \text {, and } \rho^{*}= \\
1-\frac{\zeta_{P}\left(D-d^{*}\right)^{l}}{\eta a_{r}\left(P_{T}-P_{s}^{*}\right)} \text { depend on } \zeta_{P} \text {. This leads to a tradeoff between } \\
\text { minimized } p_{\text {out }_{1}} \text { and underlying } \zeta_{P} \text {. Also, if } d^{*}=d_{J} \text {, then } \frac{P_{s}^{*}}{P_{T}}=\frac{d^{*}}{D} \text {. }\end{array}$} \\
\hline & & Objective function & jointly convex & \\
\hline & & Convex & $C 2-C 5, C 8$ & \\
\hline \multirow{3}{*}{\multicolumn{2}{|c|}{$\begin{array}{l}\text { Optimal PS (PS0) } \\
\text { common for without and } \\
\text { with direct } \mathcal{S} \text {-to- } \mathcal{D} \text { link }\end{array}$}} & Feasibility condition & $\rho^{t h} \geq 0$ & \multirow{3}{*}{$\begin{array}{l}\rho^{*}=\rho^{t h}=1-\frac{\zeta_{P}\left(\frac{D}{\epsilon}-d\right)^{l}}{\eta a_{r}\left(P_{T}-P_{s}\right)} \text { is obtained by solving } C 1 \text { at strict } \\
\text { equality. } \rho^{*} \text { and minimized outage probability are respectively } \\
\text { decreasing and increasing functions of } \zeta_{P} \text {. Also, } \widehat{p_{o u t}} \text { is convex in } \rho \text {. }\end{array}$} \\
\hline & & Objective function & pseudoconvex & \\
\hline & & Convex constraints & $C 1, C 6-C 7$ & \\
\hline$\stackrel{\underline{u}}{\Xi}$ & \multirow{2}{*}{$\begin{array}{l}\text { Optimal PA } \\
\text { (PA2) }\end{array}$} & \multicolumn{2}{|c|}{ Feasibility and constraints same as (PA1) } & \multirow{2}{*}{$\begin{array}{l}P_{s}^{*}=P_{s_{2}}^{0} \text { obtained using iterative algorithm provides lower } p_{\text {out }} \text { as } \\
\text { compared to } P_{s}^{*}=P_{s}^{t h} \text { where minimized } p_{\text {out }} \text { increases with } \zeta_{P} \text {. }\end{array}$} \\
\hline$\overbrace{0}^{1}$ & & Objective function & pseudoconvex & \\
\hline cे & \multirow{2}{*}{$\begin{array}{l}\text { Optimal RP } \\
\text { (RP2) }\end{array}$} & \multicolumn{2}{|c|}{ Feasibility and constraints same as (RP1) } & \multirow{2}{*}{$\begin{array}{l}\text { Iterative solution } d^{*}=d_{2}^{0} \text { is independent of } \zeta_{P} \text {, whereas for } d_{2}^{0}<d^{t h} \text {, } \\
\text { both } d^{*}=d^{t h} \text { and minimized } p_{\text {out }} \text { increase with increasing } \zeta_{P} \text { at } \mathcal{D} \text {. }\end{array}$} \\
\hline 苍 & & Objective function & pseudoconvex & \\
\hline ت & \multirow{2}{*}{$\begin{array}{l}\text { Joint optimization of } \\
\text { PA, RP, and PS (J2) }\end{array}$} & \multicolumn{2}{|c|}{ Feasibility and constraints same as (J1) } & \multirow{2}{*}{$\begin{array}{l}\text { Optimal solutions }\left(P_{s}^{*}, d^{*}, \rho^{*}\right) \text {, dependent on } \zeta_{P} \text {, are obtained by mini- } \\
\text { mizing } p_{o u t_{2 J}} \text { alternatively in } P_{s} \text { and } d \text {, with } \rho^{*} \text { in terms of } P_{s}^{*} \text { and } d^{*} \text {. }\end{array}$} \\
\hline 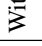 & & Objective function & multi-pseudoconvex & \\
\hline
\end{tabular}

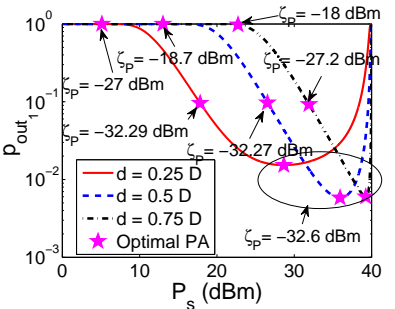

(a) Without direct $\mathcal{S}$-to- $\mathcal{D}$ link

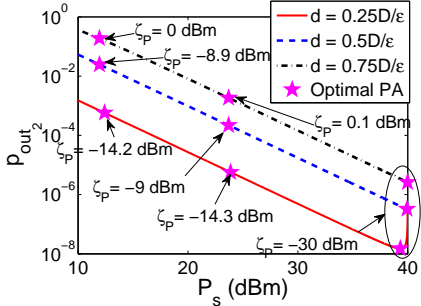

(b) With $\mathcal{S}$-to- $\mathcal{D}$ direct link

Fig. 2: Optimal PA with fixed RP and influence of minimum required harvested power $\zeta_{P}$ at $\mathcal{D}$ for $\rho=\frac{1}{2}$.

hence, it is the optimal solution. Otherwise, (J2) is infeasible.

Table II presents a summary of the main analytical results derived in Sections IV-VII. It is worth noting that the unavailability of analytical optimization solutions for direct $\mathcal{S}$-to- $\mathcal{D}$ link case (minimization of $p_{\text {out }_{2}}$ ) corroborates the importance of analytical results derived for optimal PA, RP, and PS in no direct link case (minimization of $p_{\text {out }}$ ). These analytical solutions are derived by exploiting the individual and joint convexity of $\widehat{p_{\text {out }}}$ and $\widehat{p_{\text {out }} J}$ in $P_{s}, d$, and $\rho$.

\section{NUMERICAL INVESTIGATION AND DISCUSSION}

Here we analyze the performance of the optimization schemes proposed in Sections IV-VII with the help of numerical examples. We consider $a \triangleq a_{s}=a_{r}=a_{d}=0.1$, $l=3, \zeta_{I}=10 \mathrm{bits} / \mathrm{sec} / \mathrm{Hz}$ (outage threshold), $P_{T}=40$ $\mathrm{dBm}, N_{0}=-99.85 \mathrm{dBm}, K=6 \mathrm{~dB}$ (Rice factor), $\delta=1$ m (minimum distance between $\mathcal{S}-\mathcal{R}$ or $\mathcal{R}-\mathcal{D}$ ), and $\eta=0.5$. The $\mathcal{S}$-to- $\mathcal{D}$ distance is: with direct link $D=20 \mathrm{~m}$ with $\epsilon=0.8$, and without direct link $D=100 \mathrm{~m}$ with $\epsilon=1$. Fixed (non-cooperative) allocations are assumed to be uniform, i.e., $P_{s}=P_{r}=0.5 P_{T}, d=0.5 \frac{D}{\epsilon}$, and $\rho=0.5$ (PS ratio). The tolerance for Algorithm 1 is set as $\xi=10^{-15}$.

\section{A. Optimal PA for fixed RP and PS [(PA1) and (PA2)]}

Figs. 2(a) and 2(b) illustrate the outage performance versus source power, along with optimal PA $P_{s}^{*}$ as obtained

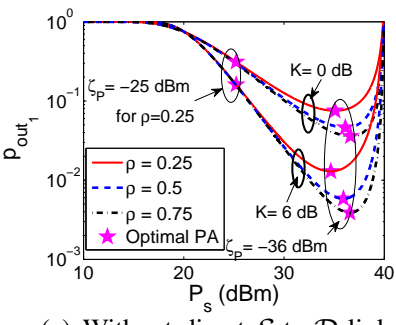

(a) Without direct $\mathcal{S}$-to- $\mathcal{D}$ link

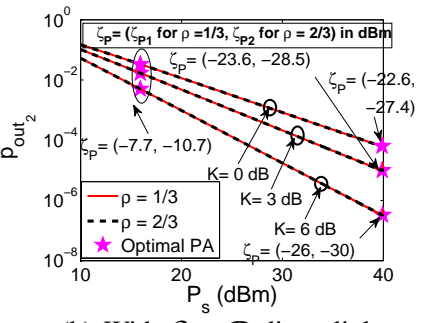

(b) With $\mathcal{S}$-to- $\mathcal{D}$ direct link
Fig. 3: Variation of $p_{\text {out }}$ with $P_{s}$ for different $\zeta_{P}, \rho$, and $K$ values with $\frac{\epsilon d}{D}=0.5$. Optimal $P_{s}^{*}$ is also plotted.

in (17) and (23), at three different relay positions: $\frac{\epsilon d}{D} \in$ $\{0.25,0.5,0.75\}$ with $\rho=0.5$. In the plots, optimal PA is shown under different harvested power requirements $\zeta_{P}$. Very low values of $\zeta_{P}<-30 \mathrm{dBm}$, have been considered to observe the performance of the proposed optimization schemes (i) with no energy harvesting requirement (that provides best outage performance) and (ii) for the applications with extremely low energy requirements. However, for most of the practical RFEH applications $\zeta_{P} \geq-20 \mathrm{dBm}$. The minimum $p_{\text {out }_{1}}$ and $p_{\text {out }_{2}}$ (in all 3 cases of RP) is achieved when $\zeta_{P}$ is very low (-32.6 dBm in Fig. 2(a) and $-30 \mathrm{dBm}$ in Fig. 2(b)) and, thus, $P_{s}^{*}=P_{s_{i}}^{0}<P_{s}^{t h} \forall i=1,2$. Also, it is shown that increasing $\zeta_{P}$ leads to increasing $p_{\text {out }_{i}} \forall i=1,2$ resulted by optimal PA as the corresponding $P_{s}^{t h}$ drops below $P_{s_{i}}^{0}$.

Remark 2: For no direct $\mathcal{S}$-to- $\mathcal{D}$ link with very low $\zeta_{P}$, minimum $p_{\text {out }_{1}}^{*}$ is achieved for $\frac{d}{D}=\frac{1}{2} \cdot \frac{d}{D}=\frac{1}{4}$ has the worst outage performance. On the other hand, $p_{\text {out }}^{*}$ increases with increased $d$. However with increasing $d$, optimal PA to $\mathcal{S}$ increases in both cases to strengthen the weakened $\mathcal{S}$-to$\mathcal{R}$ link, though this increase in direct $\mathcal{S}$-to- $\mathcal{D}$ link case is relatively lower than without direct link.

Remark 3: At very high $P_{s}$, $p_{\text {out }}$ increases with $P_{s}$ (Figs. 2(a) and 3(a)) due to weakened $\mathcal{R}$-to-D link. However, $p_{\text {out }_{2}}$ almost monotonically decreases with $P_{s}$ (Figs. 2(b) and $3(b))$ due to strengthened $\mathcal{S}$-to- $\mathcal{D}$ and $\mathcal{S}$-to- $\mathcal{R}$ links. 


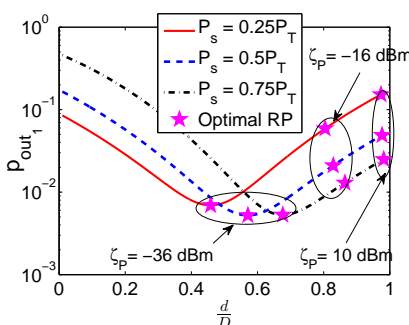

(a) Without direct $\mathcal{S}$-to- $\mathcal{D}$ link

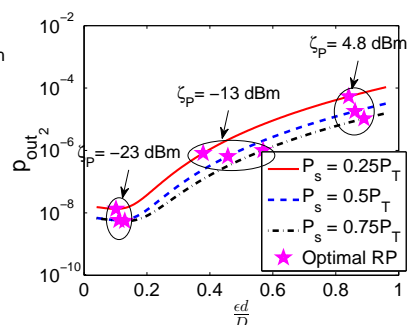

(b) With $\mathcal{S}$-to- $\mathcal{D}$ direct link
Fig. 4: Optimal RP with fixed PA and PS, along with the effect of $\zeta_{P}$ on the minimized $p_{\text {out }_{1}}, p_{\text {out }_{2}}$ and optimal RP $d^{*}$. Fixed PS considered is: $\rho=0.5$.

In Figs. 3(a) and 3(b), the variation of outage versus $P_{s}$ is plotted for different $\rho$ and $K$ values. For both without and with direct link availability, outage performance improves with increasing $\rho$ and $K$. However, for high $\zeta_{P}$, only low $\rho$ provides feasible solution (see Fig. 3(a)) because it allows higher harvested power. Also, as noted in Fig. 3(b), lower $\rho$ helps to meet higher $\zeta_{P}$.

Remark 4: Impact of $\rho$ on $p_{\text {out }}$ is almost negligible when direct link is available (Fig. 3(b)), though increased $K$ provides significantly improved outage performance in both cases.

Remark 5: The variation of $K$ has negligible impact on $P_{s}^{*}$. However, $P_{s}^{*}$ increases with increased $\rho$, though this increase is negligible for direct link case as compared to no direct link.

\section{B. Optimal RP for fixed $P A$ and $P S[(R P 1)$ and $(R P 2)]$}

Figs. 4(a) and 4(b) depict $p_{\text {out }_{i}} \forall i=1,2$ as a function of relay position for fixed PA and PS $(\rho=0.5)$, along with optimal RP $d^{*}$, as given in (28) and (32). Three different fixed PAs have been considered: $P_{s} \in\{0.25,0.5,0.75\} P_{T}$. Results in Fig. 4(a) show that $p_{\text {out }_{1}}$ achieved by optimal RP decreases with increasing $P_{s}$ and optimal RP $d^{*}$ moves closer to $\mathcal{D}$ in no direct link case to strengthen the weaker $\mathcal{R}$-to- $\mathcal{D}$ link. However, this trend is observed more clearly only at high $\zeta_{P}$ for direct link case (Fig. 4(b)). Moreover, for all values of $P_{s}, p_{\text {out }_{i}} \forall i=1,2$ due to optimal RP increases with $\zeta_{P}$ as in case of optimal PA, because increasing $d^{t h}$ goes above $d_{i}^{0}$. Minimum $p_{\text {out }}$ is achieved when $\zeta_{P}$ is very low $(-36 \mathrm{dBm}$ in Fig. 4(a) and $-23 \mathrm{dBm}$ in Fig. 4(b)) and, thus, $d^{*}=d_{i}^{0}>d^{\text {th }}$.

Remark 6: Outage performance of optimal RP for both with and without direct link is better than optimal PA, signifying that optimal RP is a better partially-adaptive scheme.

For further insight on the performance of optimal RP scheme, we plot the optimal normalized RP $\left(\frac{\epsilon d^{*}}{D}\right)$ versus relay power ratio $\left(\frac{P_{T}-P_{s}}{P_{T}}\right)$ with $\rho=0.5, K=6 \mathrm{~dB}$, and different $P_{T}$ and $\zeta_{P}$ in Figs. 5(a) and 5(b) for the two cases of $\mathcal{S}$-to- $\mathcal{D}$ link availability.

Remark 7: With higher $P_{T}$ and lower $\zeta_{P}$, optimal RP moves closer to $\mathcal{S}$ with increased $P A$ to $\mathcal{R}$ in order to have lower path loss on $\mathcal{S}$-to- $\mathcal{R}$ link. However, for higher harvested power requirements (increased $\left.\zeta_{P}\right), \mathcal{R}$ has to be positioned near $\mathcal{D}$, as shown in Figs. 5(a) and 5(b).

Remark 8: With very low $\zeta_{P}$, optimal $R P d^{*}$ is close to $\mathcal{S}$ when direct $\mathcal{S}$-to- $\mathcal{D}$ link is available (see Fig. 5(b)) and close to middle position between $\mathcal{S}$ and $\mathcal{D}$ for no direct link

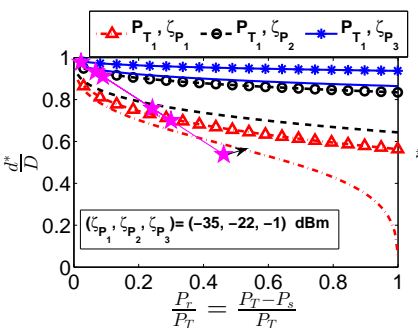

(a) Without direct $\mathcal{S}$-to- $\mathcal{D}$ link

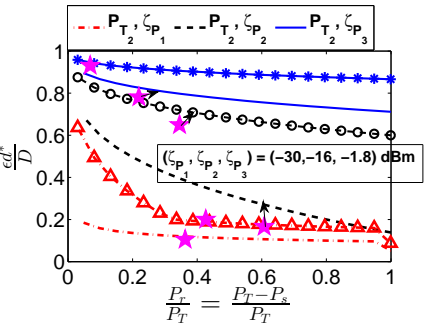

(b) With $\mathcal{S}$-to- $\mathcal{D}$ direct link
Fig. 5: Optimal normalized RP versus $\frac{P_{r}}{P_{T}}$ with $\left\{P_{T_{1}}, P_{T_{2}}\right\}$ $=\{30,40\} \mathrm{dBm}$ and $\zeta_{P}=\left\{\zeta_{P_{1}}, \zeta_{P_{2}}, \zeta_{P_{3}}\right\}$ as mentioned in respective figures. Starred points are joint-optimal solutions.

case (Fig. 5(a)). A similar trend is observed for optimal RP obtained from the joint optimization scheme. However for high $\zeta_{P}$, both optimal RP scheme and joint optimization scheme place $\mathcal{R}$ closer to $\mathcal{D}$.

\section{Optimal PS for fixed PA and RP [(PSO)]}

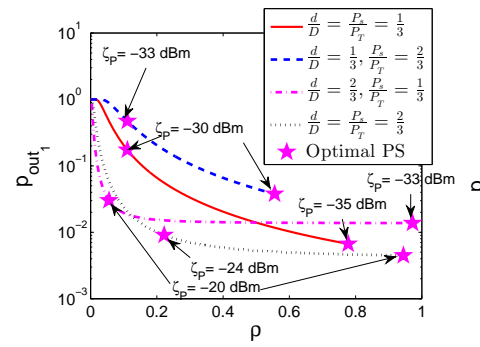

(a) Without direct $\mathcal{S}$-to- $\mathcal{D}$ link

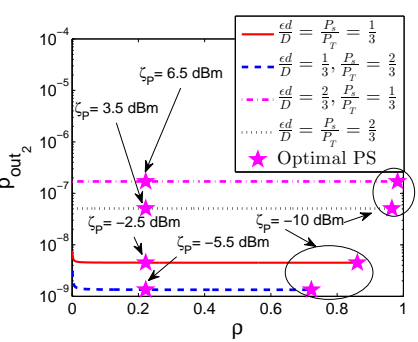

(b) With $\mathcal{S}$-to- $\mathcal{D}$ direct link
Fig. 6: Optimal PS with fixed PA and RP, and influence of $\zeta_{P}$ on outage probability and $\rho^{*}$.

Figs. 6(a) and 6(b) corroborate the monotonically decreasing behavior of $p_{\text {out }}$ with increased $\rho$ for different PA and RP values. Although this decrease in outage is significant for no direct link case (Fig. 6(a)), the decrease for direct link case is negligible. The minimized outage probability $p_{\text {out }}^{*}\left(\right.$ and $p_{\text {out }}^{*}$ ) increases and optimal PS $\rho^{*}$ decreases with increased $\zeta_{P}$.

Remark 9: For no direct link case (Fig. 6(a)), lower $P_{s}$ and higher $d$ provide lower $p_{\text {out }}$. In contrast, with $\mathcal{S}$-to-D direct link availability (Fig. $6(b))$, higher $P_{s}$ and lower d provide lower $p_{\text {out }_{2}}$. However, in both the cases, higher $d(\mathcal{R}$ closer to $\mathcal{D})$ can help to meet higher $\zeta_{P}$.

Remark 10: The optimal PS for fixed PA and RP scheme plays a more significant role in no direct link case than in the direct $\mathcal{S}$-to-D link availability case.

\section{Joint-optimal $P A, R P$, and $P S[(J 1)$ and $(J 2)]$}

The harvested power constraint $C 1$ plays a significant role in the outage performance of the joint optimization and other individual optimization schemes. Figs. 7(a) and 7(b) plot the minimized $p_{\text {out }_{i}} \forall i=1,2$ obtained by joint optimization scheme for varying $\zeta_{P}$, under different values of Rice factor $K \in\{-\infty, 0,3,6,10\} \mathrm{dB}$. Total power budget is $P_{T}=40$ $\mathrm{dBm}$. The plots are obtained by solving joint optimization problems $((\mathrm{J} 1)$ and $(\mathrm{J} 2))$ for different $\zeta_{P}$ values, one for each point on the curve. There is no feasible PA, RP, and PS for $\zeta_{P}>27 \mathrm{dBm}$ for both with and without direct link cases for all five considered values of $K$. As $\zeta_{P}$ is decreased, 


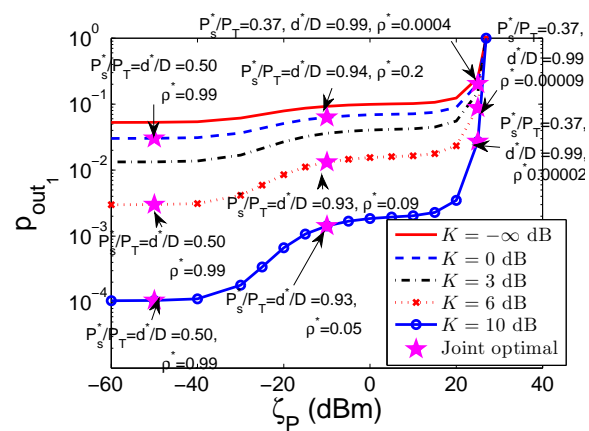

(a) Without direct $\mathcal{S}$-to- $\mathcal{D}$ link

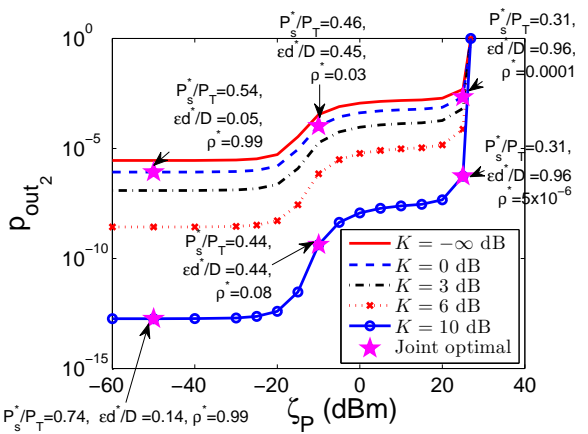

(b) With $\mathcal{S}$-to- $\mathcal{D}$ direct link

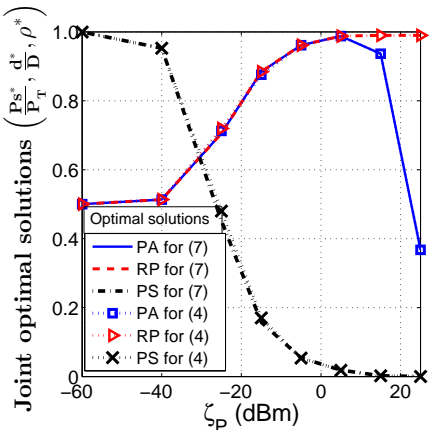

(c) Comparison of solutions for (4), (7)

Fig. 7: (a) and (b) Optimal tradeoff between the minimized outage probability provided by joint optimization scheme and lower bound $\zeta_{P}$ on required harvested power at $\mathcal{D}$. (c) Comparison of jointly-optimized solutions $\left(P_{s}^{*}, d^{*}, \rho^{*}\right)$ that minimize the exact $p_{\text {out }_{1}}$ expression (4) and its tight exponential approximation (7) with varying $\zeta_{P}$.

the minimized $p_{\text {out }}$ decreases sharply first, then more slowly until $C 1$ is no longer active. Finally, a globally Pareto-optimal tradeoff between $p_{\text {out }}$ and $\zeta_{P}$ is obtained.

The minimum $p_{\text {out }_{i}}$ is achieved when $\zeta_{P}$ is very low $(-50$ dBm in Fig. 7(a) and $-30 \mathrm{dBm}$ in Fig. 7(b)). Results show that increasing $\zeta_{P}$ beyond $-40 \mathrm{dBm}$ and $-20 \mathrm{dBm}$ for no direct link and with direct link cases, respectively, leads to an increase in minimized outage probability $p_{\text {out }_{1}}^{*}$ and $p_{\text {out }}^{*}$. Also, for the considered numerical examples, Algorithm 1 requires on an average eight iterations for converging to jointoptimal solution within acceptable tolerance.

Remark 11: Without direct $\mathcal{S}$-to-D link, the normalized optimal PA and RP increase in the same proportion with increased $\zeta_{P}$ for medium and low $\zeta_{P}$, because as $\mathcal{R}$ is moved closer to $\mathcal{D}$ to meet its $\zeta_{P}, P_{s}$ is increased to strengthen the weakened $\mathcal{S}$-to- $\mathcal{R}$ link (see Fig. $7(a)$ ).

Remark 12: For very low $\zeta_{P}$ in no direct link case, joint optimization scheme allocates equal power to $\mathcal{S}$ and $\mathcal{R}\left(\frac{P_{s}^{*}}{P_{T}} \approx\right.$ $0.5)$, and center position $\left(\frac{\epsilon d^{*}}{D} \approx 0.5\right)$ is optimal RP. However, $\rho^{*} \approx 0$ for very high $\zeta_{P}$, and $\rho^{*} \approx 1$ for very low $\zeta_{P}$ in both with and without direct link cases.

Remark 13: With direct link availability, optimal PA $P_{s}^{*}$ and PS $\rho^{*}$ provided by joint optimization scheme decreases, whereas optimal RP $d^{*}$ increases with increased $\zeta_{P}$. However, there is no trend observed for normalized proportionality of $P_{s}^{*}$ and $d^{*}$, as in case of no direct link.

Remark 14: Higher $K$ helps to achieve lower $p_{\text {out }}$, signifying that outage performance of joint optimization for SWIPT over Rician channels is better than that over Rayleigh channels. The exact optimization results for Rayleigh fading can be easily generated from our formulation, by substituting $K=0$ (which implies $\alpha_{i}=0.5$ and $\beta_{i}=1 \forall i=1,2,3$ ).

Finally, we plot jointly-optimized solutions (obtained numerically using exhaustive three-dimensional search) for minimizing exact $p_{\text {out }_{1}}$ expression (4) in Fig.7(c) and compare them with the analytical solutions derived in Section VII-A to validate the effectiveness of the derived optimal solutions.

Remark 15: Fig. 7(c) shows that the normalized jointly optimal solutions for minimizing (4) and (7) match very tightly with a minor difference of $<1 \%$. This corroborates the consideration of (5) as a tight approximation for $Q_{1}(\cdot, \cdot)$ to obtain analytical and computationally-efficient solutions for the proposed optimization schemes in SWIPT.

Remark 16: Due to the log-concavity of $Q_{1}(a, b)$ in b [41], the obtained results are not much affected by considering other tight approximations and bounds for $Q_{1}(\cdot, \cdot)$ [26], [33]. However, using (5) helps in obtaining closed-form optimal solutions for (PA1), (RPI), (PSO), and (J1).

\section{E. Outage performance comparison}

1) Without direct $\mathcal{S}$-to- $\mathcal{D}$ link: Here we present the performance comparison of the proposed optimization schemes against non-cooperative fixed (uniform) allocation scheme. The following cases are considered: (a) varying $P_{T}$ with $D=100 \mathrm{~m}$ and $K=6 \mathrm{~dB}$; (b) varying $D$ with $P_{T}=40$ $\mathrm{dBm}$ and $K=6 \mathrm{~dB}$; and (c) varying $K$ with $P_{T}=40 \mathrm{dBm}$ and $D=100 \mathrm{~m}$, for $\zeta_{P}=-25 \mathrm{dBm}$ under two different channel conditions: (i) Good: $a_{s}=a_{r}=0.5, l=2$ with noise power $=N_{0}$ and (ii) Bad: $a_{s}=a_{r}=0.1, l=3$ with noise power $=2 N_{0}$. Figs. $8(\mathrm{a}), 8(\mathrm{~b})$, and 8(c) show that $p_{\text {out }}$ resulted in each scheme reduces with increased total power $P_{T}$ budget and Rice factor $K$ values, whereas $p_{\text {out }}$ increases with increased $\mathcal{S}$-to- $\mathcal{D}$ distance $D$. The relative performances for different $P_{T}, D$, and channel conditions are captured in Fig 9. The average percentage improvement by optimal PA, optimal RP, optimal PS, and joint optimization schemes over the fixed allocation scheme are respectively around $3.86 \%$, $12.61 \%, 36.18 \%$, and $36.31 \%$, in Good channel conditions, and around $10.15 \%, 19.45 \%, 30.68 \%$, and $42.33 \%$ in $\mathrm{Bad}$ channel conditions (when $p_{\text {out }}<1$ for fixed allocation scheme). The imposition of $C 1$ increases the significance of optimal PA, RP, and PS, because fixed allocation sometimes cannot provide a feasible solution as shown in Figs. 8(a) $\left(P_{T} \leq\right.$ $42 \mathrm{dBm}), 8(\mathrm{~b})$, and 8(c) for Bad channel conditions ( $p_{\text {out }}$ for infeasible case is plotted as 1). Even optimal PA and optimal PS schemes are infeasible at lower values of $P_{T}$ and higher values of $D$. Due to this, in Bad channel conditions when fixed allocation scheme cannot meet $\zeta_{P}$ and hence $p_{\text {out }}=1$ due to its infeasibility, the proposed optimization schemes provide much higher outage improvement. The average percentage improvement by optimal PA, optimal RP, optimal PS, and joint optimization schemes over the fixed allocation scheme with $p_{\text {out }}=1$ (infeasibility of fixed allocation scheme) are respectively around $27.25 \%, 89.42 \%, 23.55 \%$, and $92.0 \%$. 


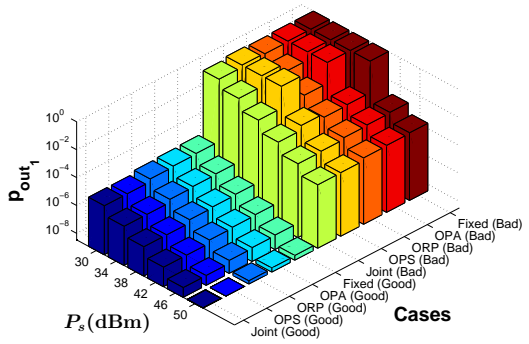

(a) $p_{\text {out }}$ variation with $P_{T}$

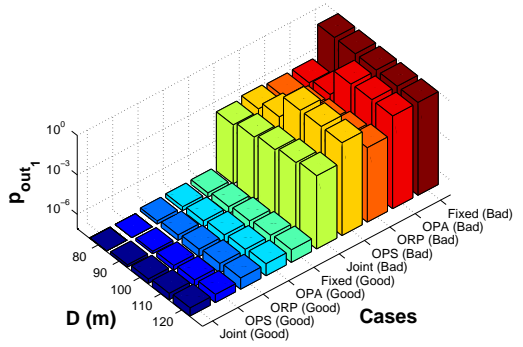

(b) $p_{\text {out }_{1}}$ variation with $D$

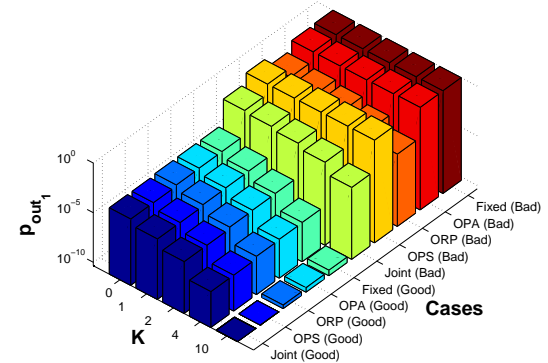

(c) $p_{\text {out }}$ variation with $K$

Fig. 8: Outage performance comparison of fixed allocation, optimal PA (OPA), optimal RP (ORP), optimal PS (OPS), and joint optimization schemes under Good and Bad channel conditions for no direct link case.

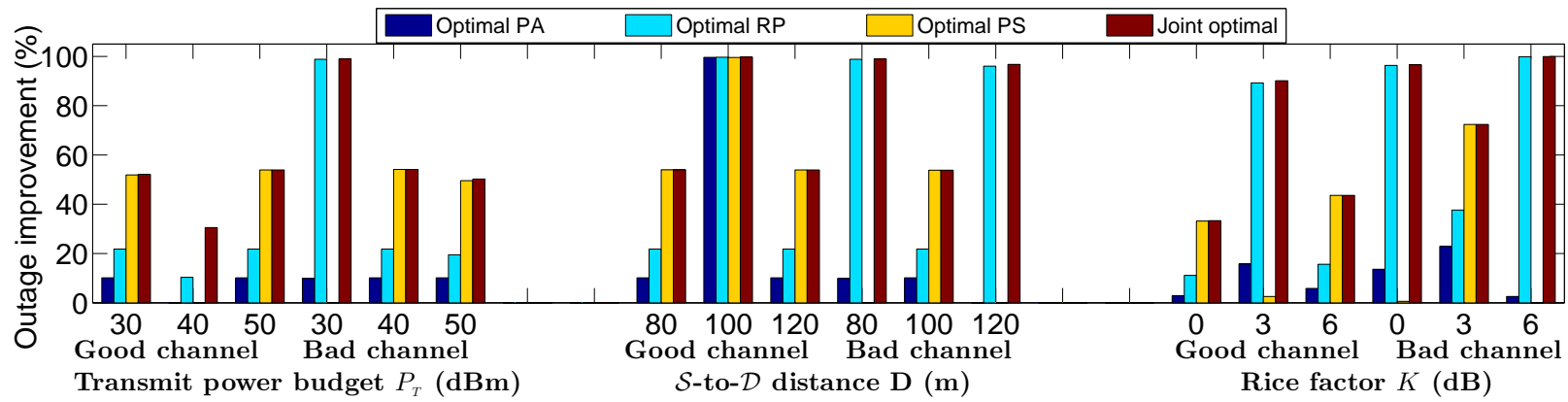

Fig. 9: Performance comparison of the proposed optimization schemes for the no direct link case with fixed allocation scheme for different total power budget $P_{T}, \mathcal{S}$-to- $\mathcal{D}$ distance $D$, and Rice factor $K$.

Remark 17: Impact of the proposed optimization schemes is much more significant when there is no direct link availability because non-cooperative fixed allocation scheme for SWIPT mostly suffers from total outage even for low energy requirements $\left(\zeta_{P} \approx-25 \mathrm{dBm}\right)$ at $\mathcal{D}$.

Remark 18: Joint optimization scheme always performs the best because it has the highest degree of freedom. The outage performance order (best to worst) is: \{joint-optimal, optimal PS, optimal RP, optimal PA\} for low $\zeta_{P}$ or good channel conditions, and \{joint-optimal, optimal RP, optimal $P A$, optimal $P S\}$ for high $\zeta_{P}$ or bad channel conditions.

2) With $\mathcal{S}$-to-D direct link available: A similar comparison is performed when direct $\mathcal{S}$-to- $\mathcal{D}$ link is available, with $\zeta_{P}=0$ $\mathrm{dBm}$. The variations of the following four parameters are studied: (a) transmit power budget $P_{T}$; (b) $\mathcal{S}$-to- $\mathcal{D}$ distance $D$; (c) channel conditions; (d) Rice factor $K$ (see Fig. 10). Under each variation other three parameters are respectively kept fixed as $P_{T}=40 \mathrm{dBm}, D=20 \mathrm{~m}, K=6 \mathrm{~dB}$, and Good channel conditions $\left(a=0.5, l=2, N_{0}=-99.85\right.$ $\mathrm{dBm})$. The channel deterioration is implemented by decreasing the channel gains $\left(a_{s}, a_{r}\right.$, and $\left.a_{d}\right)$ and increasing path loss exponent $l$ and noise power $N_{0}$ with respect to the Good channel conditions (0\% deterioration). Figs. 10(a) and 10(d) show that $p_{\text {out }_{2}}$ resulted in each scheme reduces with increased $P_{T}$ and $K$, respectively. Instead, Figs. 10(b) and 10(c) show that $p_{\text {out }}$ increases with increased $\mathcal{S}$-to- $\mathcal{D}$ distance $D$ and channel deterioration, respectively. In all four variations, jointoptimal PA, RP, and PS has the best outage performance, closely followed by optimal RP with fixed PA and PS. Optimal PA with fixed RP and PS also provides significant outage performance improvement, except being infeasible in certain cases, like very low $P_{T}$ and very poor channel conditions.
However, optimal PS does not provide any practical improvement, except in the case when the fixed allocation scheme results in an infeasible solution (i.e., $p_{\text {out }_{2}}=1$ ).

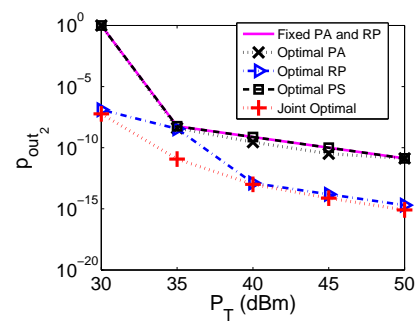

(a) Variation with $P_{T}$

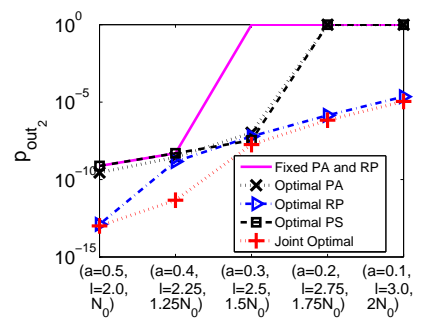

(c) Variation with $a, l, N_{0}$

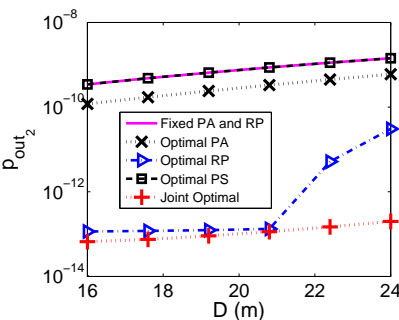

(b) Variation with $D$

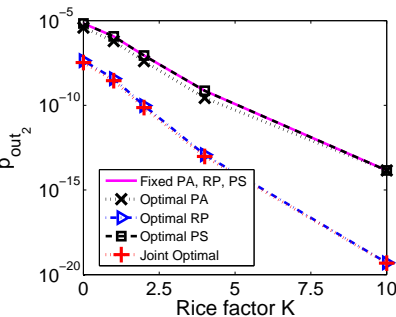

(d) Variation with $K$
Fig. 10: Outage $p_{\text {out }}$ performance comparison of the proposed optimization schemes with fixed allocation scheme.

The relative performance results for direct $\mathcal{S}$-to- $\mathcal{D}$ link case are summarized in Fig 11. The average outage performance improvement by optimal PA, optimal RP, optimal PS, and joint optimization schemes over fixed allocation scheme (when it provides a feasible solution, i.e., pout $_{2}<1$ ), are respectively around $43.62 \%, 99.35 \%, 7.36 \times 10^{-4 \%}, 99.55 \%$.

Remark 19: The proposed optimization schemes for efficient SWIPT outperform the fixed allocation, which suffers from 


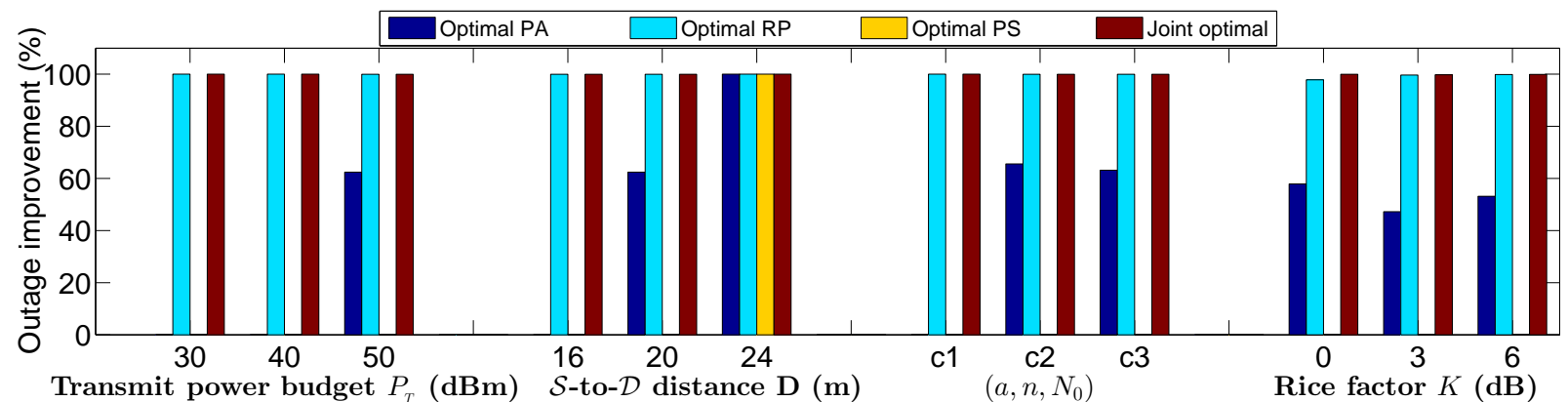

Fig. 11: Performance comparison of the proposed optimization schemes against fixed allocation for direct link case. The percentage improvement results are with respect to $p_{\text {out }}$ resulted from fixed allocation scheme. Channel condition is varied as: $\mathrm{c} 1=\left(0.5,2, N_{0}\right)$ (Good channel), $\mathrm{c} 2=\left(0.3,2.5,1.5 N_{0}\right)$, and $\mathrm{c} 3=\left(0.1,3,2 N_{0}\right)$ (Bad channel).

total outage while meeting energy requirements at $\mathcal{D}$ for low $P_{T}$, high $D$, and poor channel conditions. This efficiency improvement provided by optimal PA, optimal RP, and jointoptimal schemes is much more evident and significant in case of direct $\mathcal{S}$-to-D link availability (Fig 11) as compared to the no direct link case (Fig 9).

Remark 20: The optimal RP scheme performs much better than optimal PA and optimal PS schemes, and closely follows the best outage performance provided by the jointly optimized scheme for direct link case. However, the scenarios where due to terrain asperities/blockage, $\mathcal{R}$ has to be placed at some specific position, optimal PA is the only efficient optimization scheme possible, because optimal PS does not provide much improvement over fixed allocation scheme.

\section{CONCLUding Remarks}

This paper has investigated the optimization of PA, RP, and PS in two-hop information relaying and energy transfer to minimize the outage probability in SWIPT over Rician channels, subject to total transmit power and harvested power constraints. Two scenarios of source-to-destination $(\mathcal{S}$-to- $\mathcal{D})$ distances, direct $\mathcal{S}$-to- $\mathcal{D}$ link availability and no direct $\mathcal{S}$ to- $\mathcal{D}$ link have been considered. Analytical expressions have been obtained for the four optimization schemes: optimal PA, optimal RP, optimal PS, and joint optimization of PA, RP, and PS for no direct link scenario. In direct link case, joint global optimal PA, RP, and PS has been derived by exploiting multipseudoconvexity of outage probability and using an alternating optimization based iterative scheme. Numerical results show that, in general, the joint optimization scheme performs the best, respectively followed by optimal RP and optimal PA. The results also highlight that the direct link case has different outage performance under the four optimization schemes as compared to the case without direct link. For low required harvested power at $\mathcal{D}$ without direct $\mathcal{S}$-to- $\mathcal{D}$ link, optimal RP is close to the center position between $\mathcal{S}$ and $\mathcal{D}$, whereas when direct link is available, optimal RP is closer to $\mathcal{S}$ with higher PA to $\mathcal{S}$. With increasing required harvested power, the normalized optimal PA and RP increase in same proportion in no direct link scenario, whereas no such trend is observed when direct link is available. Also, in general, the optimal PS ratio in no direct link scenario is relatively higher as compared to when direct link is available. The performance of all four optimization schemes are strongly influenced by the required harvested power due to the tradeoff between the minimized outage probability and the required harvested power at $\mathcal{D}$.

\section{APPENDIX A \\ INDIVIDUAL PSEUDOCONVEXITY OF OUTAGE Probability $p_{\text {out }_{2}}$ IN SOURCE Power $P_{s}$}

Here we provide proof of Lemma 1 and the claims made in Theorem 2. In this regard, we first propose Lemma 5 which will be used in proving tri-pseudoconvexity of $p_{\text {out }}$ in $P_{s}, d, \rho$.

Lemma 5: A positive differentiable function $f$, which is $\log$ concave over a convex set $\mathbb{F}$, is also pseudoconcave over $\mathbb{F}$.

Proof: A function $f$ is concave if and only if $\mathbb{F}$ is convex and it satisfies (A.1) [42].

$$
f(y) \leq f(x)+\nabla f(x)^{\top}(y-x) \quad \forall x, y \in \mathbb{F} .
$$

A similar relationship for a positive differentiable log-concave function $f$ is given by (A.2).

$$
\log f(y) \leq \log f(x)+\nabla \log f(x)^{\top}(y-x) \quad \forall x, y \in \mathbb{F} .
$$

A function $f$ is pseudoconcave if $-f$ is pseudoconvex, or $\forall x, y \in \mathbb{F}$ (a convex set), $\nabla f(x)^{\top}(y-x) \leq 0 \Longrightarrow$ $f(x)-f(y) \geq 0$. Applying $\nabla f(x)^{\top}(y-x) \leq 0$ in (A.2) and using the positivity of $f(x)$, we obtain: $\log f(y) \leq$ $\log f(x)+\frac{1}{f(x)} \nabla f(x)^{\top}(y-x) \leq \log f(x)$, which on using the monotonicity of $\log (\cdot)$ implies $f(y) \leq f(x)$. This proves that $\log$-concavity of a positive differentiable function $f$ also implies the pseudoconcavity of $f$.

\section{A. Proof of Lemma 1: $p_{\text {out }_{2}}$ is pseudoconvex in $P_{s}$ for fixed $R P$ and $P S$}

First of all we recall a very important property, which states that, for a single variable function the concepts of pseudoconcavity and unimodality are completely equivalent [43]. Using this property, if we show that a function is unimodal in $P_{s}$ over the feasible region defined by $C 1-C 3$, it also implicitly implies its pseudoconcavity in $P_{s}$. Using $p_{\text {out }_{2}}=$ $1-\mathcal{C}_{\gamma_{1}}(\mathcal{Z}) \mathcal{C}_{\Upsilon_{02}}(\mathcal{Z})$, we obtain:

$$
\begin{aligned}
p_{\text {out }_{2}} & =1+\mathcal{C}_{\gamma_{1}}(\mathcal{Z}) \int_{\mathcal{Z}}^{\infty}\left(\frac{\mathrm{d} F_{\gamma_{0}}(x)}{\mathrm{d} x}\right)\left(\frac{\mathrm{d} F_{\gamma_{2}}\left(\frac{\mathcal{Z}-x}{\rho}\right)}{\mathrm{d} x}\right) \mathrm{d} x \\
& =1-\mathcal{C}_{\gamma_{1}}(\mathcal{Z}) \int_{\mathcal{Z}}^{\infty} \mathcal{G}_{1}\left(P_{s}, d, \rho, x\right) \mathrm{d} x .
\end{aligned}
$$


Here $\mathcal{G}_{1}\left(P_{s}, d, \rho, x\right) \triangleq\left[\frac{\alpha_{0}^{\prime} \beta_{0} \alpha_{2}^{\prime \prime} \beta_{2}}{x(\mathcal{Z}-x)}\left(\frac{D^{l}}{a_{d} P_{s}}\right)^{\beta_{0}}\left(\frac{\left(\frac{D}{\epsilon}-d\right)^{l}}{\rho a_{r}\left(P_{T}-P_{s}\right)}\right)^{\beta_{2}} \times\right.$ $\left.e^{-\alpha_{0}^{\prime}\left(\frac{D^{l}}{a_{d} P_{s}}\right)^{\beta_{0}}-\alpha_{2}^{\prime \prime}\left(\frac{\left(\frac{D}{\epsilon}-d\right)^{l}}{\rho a_{r}\left(P_{T}-P_{s}\right)}\right)^{\beta_{2}}}\right], \alpha_{0}^{\prime}=e^{\phi\left(\sqrt{2 K_{0}}\right)}\left(2\left(K_{0}+1\right) N_{0} x\right)^{\beta_{0}}$ and $\alpha_{2}^{\prime \prime}=e^{\phi\left(\sqrt{2 K_{2}}\right)}\left(2\left(K_{2}+1\right) N_{0}(\mathcal{Z}-x)\right)^{\beta_{2}}$.

Next we find the critical point of $\mathcal{G}_{1}$ in $P_{s}$ by solving $\frac{\partial \mathcal{G}_{1}}{\partial P_{s}}=0$. Except the trivial case with $\alpha_{0}^{\prime}\left(\frac{D^{l}}{a_{d} P_{s}}\right)^{\beta_{0}}=1$ and $\alpha_{2}^{\prime \prime}\left(\frac{\left(\frac{D}{\epsilon}-d\right)^{l}}{\rho a_{r}\left(P_{T}-P_{s}\right)}\right)^{\beta_{2}}=1$, critical point of $\mathcal{G}_{1}$ is obtained by numerically solving (A.4). Let the solution of (A.4) be $\overline{P_{s}}$. Now if $\alpha_{0}^{\prime}\left(\frac{D^{l}}{a_{d} P_{s}}\right)^{\beta_{0}}>1$ and $\alpha_{2}^{\prime \prime}\left(\frac{\left(\frac{D}{\epsilon}-d\right)^{l}}{\rho a_{r}\left(P_{T}-P_{s}\right)}\right)^{\beta_{2}}>1$, $\mathcal{G}_{1}$ is respectively an increasing and a decreasing function of $P_{s}$ for $P_{s}<\overline{P_{s}}$ and $P_{s}>\overline{P_{s}}$. This behavior gets reversed if $\alpha_{0}^{\prime}\left(\frac{D^{l}}{a_{d} P_{s}}\right)^{\beta_{0}}<1$ and $\alpha_{2}^{\prime \prime}\left(\frac{\left(\frac{D}{\epsilon}-d\right)^{l}}{\rho a_{r}\left(P_{T}-P_{s}\right)}\right)^{\beta_{2}}<1$. This proves that $\mathcal{G}_{1}$ is a pseudoconcave function [43] of $P_{s}$. As integration preserves the pseudoconcavity of positive pseudoconcave function [44], pseudoconcavity of $\mathcal{G}_{1}$ in $P_{s}$ also implies pseudoconcavity of $\mathcal{C}_{\Upsilon_{02}}(\mathcal{Z})=\int_{\mathcal{Z}}^{\infty} \mathcal{G}_{1} \mathrm{~d} x$. Apart from this, since $\frac{\partial^{2} \log \left[\mathcal{C}_{\gamma_{1}}(\mathcal{Z})\right]}{\partial P_{s}^{2}}=-\frac{\alpha_{1} \beta_{1}\left(\beta_{1}+1\right) d^{\beta_{1} l}}{a_{s}^{\beta_{1}} P_{s}^{\beta_{1}+2}}<0 \forall P_{s}>0$, it implies log-concavity, and hence pseudoconcavity of $\mathcal{C}_{\gamma_{1}}$ in $P_{s}$ on applying Lemma 5. Also, it may be noted that the product of two positive pseudoconcave functions is also pseudoconcave [43]. Hence, $\mathcal{C}_{\gamma_{1}}(\mathcal{Z}) \mathcal{C}_{\Upsilon_{02}}(\mathcal{Z})$ is pseudoconcave in $P_{s}$, which implies pseudoconvexity of $p_{\text {out }}$ in $P_{s}$.

\section{B. $p_{\text {out }}$ is is pseudoconvex in $P_{s}$ for fixed $R P$}

As $p_{\text {out }}$ can be obtained from $p_{\text {out }}$ by substituting $\rho=$ $1-\frac{\zeta_{P}\left(\frac{D}{\epsilon}-d\right)^{l}}{\eta a_{r}\left(P_{T}-P_{s}\right)}$, the proof of pseudoconvexity of $p_{\text {out }}$ in $P_{s}$ is similar as the proof of pseudoconvexity of $p_{\text {out }}$.

$p_{\text {out }_{2 J}}=1-\mathcal{C}_{\gamma_{1}}(\mathcal{Z})\left[1-\int_{0}^{\mathcal{Z}} \frac{\mathrm{d} F_{\gamma_{0}}(x)}{\mathrm{d} x} F_{\gamma_{2}}\left(\frac{\eta a_{r}\left(P_{T}-P_{s}\right)(\mathcal{Z}-x)}{\eta a_{r}\left(P_{T}-P_{s}\right)-\zeta_{P}\left(\frac{D}{\epsilon}-d\right)^{l}}\right) \mathrm{d} x\right]$

$$
=1-\mathcal{C}_{\gamma_{1}}(\mathcal{Z}) \int_{\mathcal{Z}}^{\infty} \widehat{\mathcal{G}_{1}}\left(P_{s}, d, x\right) \mathrm{d} x
$$

with $\widehat{\mathcal{G}_{1}}\left(P_{s}, d, x\right) \triangleq \frac{\mathcal{Z}}{\alpha_{0}^{\prime} \beta_{0} \alpha_{2}^{\prime \prime} \beta_{2}}\left(\frac{D^{l}}{x(\mathcal{Z}-x)}\right)^{\beta_{0}}\left(\frac{\eta\left(\frac{D}{\epsilon}-d\right)^{l}}{\eta a_{r}\left(P_{T}-P_{s}\right)-\zeta_{P}\left(\frac{D}{\epsilon}-d\right)^{l}}\right)^{\beta_{2}}$

$\times e^{-\alpha_{0}^{\prime}\left(\frac{D^{l}}{a_{d} P_{s}}\right)^{\beta_{0}}-\alpha_{2}^{\prime \prime}\left(\frac{\eta\left(\frac{D}{\epsilon}-d\right)^{l}}{\eta a_{r}\left(P_{T}-P_{s}\right)-\zeta_{P}\left(\frac{D}{\epsilon}-d\right)^{l}}\right)^{\beta_{2}}}$. The critical point of $\widehat{\mathcal{G}_{1}}$ in $P_{s}$ (i.e., $\frac{\partial \widehat{\mathcal{G}_{1}}}{\partial P_{s}}=0$ ) is obtained by numerically solving (A.6). Let the solution of (A.6) be $\widehat{P_{s}}$. Now, similar to the observation in Appendix A-A, if $\alpha_{0}^{\prime}\left(\frac{D^{l}}{a_{d} P_{s}}\right)^{\beta_{0}}>1$ and $\alpha_{2}^{\prime \prime}\left(\frac{\eta\left(\frac{D}{\epsilon}-d\right)^{l}}{\eta a_{r}\left(P_{T}-P_{s}\right)-\zeta_{P}\left(\frac{D}{\epsilon}-d\right)^{l}}\right)^{\beta_{2}}>1, \widehat{\mathcal{G}_{1}}$ is respectively an increasing and a decreasing function of $P_{s}$ for $P_{s}<\widehat{P}_{s}$ and $P_{s}>\widehat{P_{s}}$. The function behavior gets reversed for $\alpha_{0}^{\prime}\left(\frac{D^{l}}{a_{d} P_{s}}\right)^{\beta_{0}}<1$ and $\alpha_{2}^{\prime \prime}\left(\frac{\eta\left(\frac{D}{\epsilon}-d\right)^{l}}{\eta a_{r}\left(P_{T}-P_{s}\right)-\zeta_{P}\left(\frac{D}{\epsilon}-d\right)^{l}}\right)^{\beta_{2}}<1$. This proves that $\widehat{\mathcal{G}_{1}}$ is a pseudoconcave function of $P_{s}$. This along with the discussions related to the integration and product of positive pseudoconcave functions, as mentioned in Appendix A-A, proves pseuodoconvexity of $p_{o u t_{2 J}}$ in $P_{s}$.

\section{APPENDIX B \\ INDIVIDUAL PSEUDOCONVEXITY OF OUTAGE PROBABILITY $p_{\text {out }}$ IN $\mathcal{S}$-TO- $\mathcal{R}$ DISTANCE $d$}

This appendix provides proofs for Lemma 3 and the claims

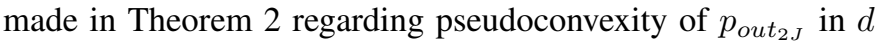
for fixed PA. We note that, $p_{\text {out }_{2}}$ in (A.3) can be rewritten as:

$$
\begin{aligned}
p_{\text {out }_{2}}= & 1-\mathcal{C}_{\gamma_{1}}(\mathcal{Z}) \mathcal{C}_{\Upsilon_{02}}(\mathcal{Z})=1-\mathcal{C}_{\gamma_{1}}(\mathcal{Z})\left[\mathcal{C}_{\gamma_{0}}(\mathcal{Z})\right. \\
& \left.+\int_{0}^{\mathcal{Z}} \frac{\mathrm{d} F_{\gamma_{0}}(x)}{\mathrm{d} x} \mathcal{C}_{\gamma_{2}}\left(\frac{\mathcal{Z}-x}{\rho}\right) \mathrm{d} x\right] . \\
\frac{\partial^{2} \log \left[\mathcal{C}_{\gamma_{1}}(\mathcal{Z})\right]}{\partial d^{2}}= & -\frac{\alpha_{1} \beta_{1} l\left(\beta_{1} l-1\right) d^{\beta_{1} l-2}}{\left(a_{s} P_{s}\right)^{\beta_{1}}}<0 \forall(d>0) \wedge(l>1)
\end{aligned}
$$
implies $\log$-concavity of $d$ in $\mathcal{C}_{\gamma_{1}}(\mathcal{Z})$.

A. Proof of Lemma 3: $p_{\text {out }_{2}}$ is pseudoconvex in $d$ for fixed PA and $P S$

$$
\begin{gathered}
\text { Let } \mathcal{G}_{2}\left(P_{s}, d, \rho, x\right) \triangleq \frac{\mathrm{d} F_{\gamma_{0}}(x)}{\mathrm{d} x} \mathcal{C}_{\gamma_{2}}\left(\frac{\mathcal{Z}-x}{\rho}\right)=\frac{\alpha_{0}^{\prime} \beta_{0}}{x}\left(\frac{D^{l}}{a_{d} P_{s}}\right)^{\beta_{0}} \\
e^{-\alpha_{0}^{\prime}\left(\frac{D^{l}}{a_{d} P_{s}}\right)^{\beta_{0}}-\alpha_{2}^{\prime \prime}\left(\frac{\left(\frac{D}{\epsilon}-d\right)^{l}}{\rho a_{r}\left(P_{T}-P_{s}\right)}\right)^{\beta_{2}}} \text {. Using } \mathcal{G}_{2} \text { definition in (B.1), } \\
\mathcal{C}_{\Upsilon_{02}}(\mathcal{Z})=\mathcal{C}_{\gamma_{0}}(\mathcal{Z})+\int_{0}^{\mathcal{Z}} \mathcal{G}_{2}\left(P_{s}, d, \rho, x\right) \mathrm{d} x . \\
\frac{\partial^{2} \log \mathcal{G}_{2}}{\partial d^{2}}=-\frac{\alpha_{2}^{\prime \prime} \beta_{2} l\left(\beta_{2} l-1\right)\left(\frac{D}{\epsilon}-d\right)^{\beta_{2} l-2}}{\left(\rho a_{r}\left(P_{T}-P_{s}\right)\right)^{\beta_{2}}}<0 \text { implies that } \mathcal{G}_{2}
\end{gathered}
$$
is a log-concave function of $d$. Since the log-concavity of a positive function is preserved under integration [42], [45], we note that $\int_{0}^{\mathcal{Z}} \mathcal{G}_{2}\left(P_{s}, d, \rho, x\right) \mathrm{d} x$ is also log-concave in $d$. Since $\mathcal{C}_{\gamma_{0}}(\mathcal{Z})=e^{-\alpha_{0}\left(\frac{D^{l}}{a_{d} P_{s}}\right)^{\beta_{0}}}$ is independent of $d$ and logconcavity is preserved under affine transformation, $\mathcal{C}_{\Upsilon_{02}}(\mathcal{Z})$ is log-concave in $d$. As the product of two log-concave functions is also log-concave [42], it results in log-concavity of $\mathcal{C}_{\gamma_{1}}(\mathcal{Z}) \mathcal{C}_{\Upsilon_{02}}(\mathcal{Z})$, which by using Lemma 5 implies pseudoconvexity of $p_{\text {out }_{2}}$ in $d$.

$$
\begin{gathered}
P_{T}\left[-1+\alpha_{0}^{\prime}\left(\frac{D^{l}}{a_{d} P_{s}}\right)^{\beta_{0}}\right]=P_{s}\left[-2+\alpha_{0}^{\prime}\left(\frac{D^{l}}{a_{d} P_{s}}\right)^{\beta_{0}}+\alpha_{2}^{\prime \prime}\left(\frac{\left(\frac{D}{\epsilon}-d\right)^{l}}{\rho a_{r}\left(P_{T}-P_{s}\right)}\right)^{\beta_{2}}\right] . \\
\left(\alpha_{0}^{\prime}\left(\frac{D^{l}}{a_{d} P_{s}}\right)^{\beta_{0}}-1\right)\left(\eta a_{r}\left(P_{T}-P_{s}\right)-\zeta_{P}\left(\frac{D}{\epsilon}-d\right)^{l}\right)=\frac{\beta_{2}}{\beta_{0}} \eta a_{r} P_{s}\left(\alpha_{2}^{\prime \prime}\left(\frac{\eta\left(\frac{D}{\epsilon}-d\right)^{l}}{\eta a_{r}\left(P_{T}-P_{s}\right)-\zeta_{P}\left(\frac{D}{\epsilon}-d\right)^{l}}\right)^{\beta_{2}}-1\right)
\end{gathered}
$$




\section{B. $p_{\text {out }_{2 J}}$ is pseudoconvex in $d$ for fixed $P A$}

$p_{\text {out }_{2 J}}$ in terms of $F_{\gamma_{0}}, \mathcal{C}_{\gamma_{0}}, \mathcal{C}_{\gamma_{1}}$, and $\mathcal{C}_{\gamma_{2}}$ is expressed as:

$$
\begin{aligned}
p_{\text {out }_{2 J}=}= & 1-\mathcal{C}_{\gamma_{1}}(\mathcal{Z})\left[\mathcal{C}_{\gamma_{0}}(\mathcal{Z})+\int_{0}^{\mathcal{Z}} \frac{\mathrm{d} F_{\gamma_{0}}(x)}{\mathrm{d} x}\right. \\
& \left.\mathcal{C}_{\gamma_{2}}\left(\frac{\eta a_{r}\left(P_{T}-P_{s}\right)(\mathcal{Z}-x)}{\eta a_{r}\left(P_{T}-P_{s}\right)-\zeta_{P}\left(\frac{D}{\epsilon}-d\right)^{l}}\right) \mathrm{d} x\right]
\end{aligned}
$$

Let $\widehat{\mathcal{G}_{2}}\left(P_{s}, d, \rho, x\right) \triangleq \frac{\mathrm{d} F_{\gamma_{0}}(x)}{\mathrm{d} x} \mathcal{C}_{\gamma_{2}}\left(\frac{\mathcal{Z}-x}{\rho}\right)=\frac{\alpha_{0}^{\prime} \beta_{0}}{x}\left(\frac{D^{l}}{a_{d} P_{s}}\right)^{\beta_{0}}$ $e^{-\alpha_{0}^{\prime}\left(\frac{D^{l}}{a_{d} P_{s}}\right)^{\beta_{0}}-\alpha_{2}^{\prime \prime}\left(\frac{\eta\left(\frac{D}{\epsilon}-d\right)^{l}}{\eta a_{r}\left(P_{T}-P_{s}\right)-\zeta_{P}\left(\frac{D}{\epsilon}-d\right)^{l}}\right)^{\beta_{2}}}$ $-\frac{\eta^{\beta_{2}+1}\left(\beta_{2} l-1\right) a_{r}\left(P_{T}-P_{s}\right)-\zeta_{P}(l+1)\left(\frac{D}{\epsilon}-d\right)^{l}\left[\alpha_{2}^{\prime \prime} \beta_{2} \eta l a_{r}\left(P_{T}-P_{s}\right)\right]}{\left(\frac{D}{\epsilon}-d\right)^{2-\beta_{2} l}\left[\eta a_{r}\left(P_{T}-P_{s}\right)-\zeta_{P}\left(\frac{D}{\epsilon}-d\right)^{l}\right]^{\beta_{2}+2}}<$ $0 \forall\left\{d \mid\left(d \leq \frac{D}{\epsilon}\right) \wedge\left(P_{s} \leq P_{T}\right) \wedge(l \geq 1) \wedge\left(\frac{\zeta_{P}\left(\frac{D}{\epsilon}-d\right)^{l}}{\eta a_{r}\left(P_{T}-P_{s}\right)} \leq 1\right)\right\}$. Similar to Appendix B-A, using Lemma 5, independence of $\mathcal{C}_{\gamma_{0}}$ in $d$, log-concavity preservation properties of affine transformation, integration, and product of log-concave functions, it is inferred that $p_{\text {out }}$ JJ is pseudoconvex in $d$.

\section{APPENDIX C}

Proof of Lemma 4: OUtAge Probability $p_{\text {out }}$ IS PSEUDOCONVEX IN PS RATIO $\rho$

To show pseudoconvexity of $p_{\text {out }_{2}}=1-\mathcal{C}_{\gamma_{1}}(\mathcal{Z}) \mathcal{C}_{\Upsilon_{02}}(\mathcal{Z})$ where $\mathcal{C}_{\Upsilon_{02}}(\mathcal{Z})$ defined in (B.2), we first prove $\log$ concavity of $\mathcal{G}_{2}$ in $\rho$, followed by the pseudoconcavity of $\mathcal{C}_{\gamma_{1}}(\mathcal{Z}) \mathcal{C}_{\Upsilon_{02}}(\mathcal{Z})$ in $\rho$. On taking second order derivative of $\mathcal{G}_{2}$ with respect to $\rho, \frac{\partial^{2} \log \mathcal{G}_{2}}{\partial \rho^{2}}=-\frac{\alpha_{2}^{\prime \prime} \beta_{2}\left(\beta_{2}+1\right)}{\rho^{2}}\left[\frac{\left(\frac{D}{\epsilon}-d\right)^{l}}{\rho a_{r}\left(P_{T}-P_{s}\right)}\right]^{\beta_{2}}<$ $0 \forall\left\{\rho \mid(\rho>0) \wedge\left(d \leq \frac{D}{\epsilon}\right) \wedge\left(P_{s} \leq P_{T}\right)\right\}$ implies that $\mathcal{G}_{2}$ is a log-concave function of $\rho$. Since $\mathcal{C}_{\gamma_{0}}(\mathcal{Z})$ and $\mathcal{C}_{\gamma_{1}}(\mathcal{Z})$ are independent of $\rho$, and log-concavity is preserved under affine transformation [42], integration [45], and positive scalar multiplication, it can be inferred from (B.1) and (B.2) that $\mathcal{C}_{\gamma_{1}}(\mathcal{Z}) \mathcal{C}_{\Upsilon_{02}}(\mathcal{Z})$ is log-concave in $\rho$. This, by using Lemma 5 , also implies that $\mathcal{C}_{\gamma_{1}}(\mathcal{Z}) \mathcal{C}_{\Upsilon_{02}}(\mathcal{Z})$ is pseudoconcave in $\rho$. Hence, $p_{\text {out }_{2}}$ is pseudoconvex in $\rho$.

\section{APPENDIX D}

Proof of JoInt Convexity of ApProXimated OUtAGE PROBABILITY $\widehat{p_{\text {out }_{1 J}}}$ WITH $\rho=\rho^{\text {th }}$

$\widehat{p_{\text {out }} \text { IJ }}$ can be represented as a sum of two functions $g_{1}=$ $\alpha_{1}\left(\frac{d^{l}}{a_{s} P_{s}}\right)^{\beta_{1}}$ and $g_{2}=\alpha_{2}\left(\frac{\eta\left(\frac{D}{\epsilon}-d\right)^{l}}{\eta a_{r}\left(P_{T}-P_{s}\right)-\zeta_{P}\left(\frac{D}{\epsilon}-d\right)^{l}}\right)^{\beta_{2}}$. Joint convexity of $\widehat{p_{\text {out } 1 J}}$ in $P_{s}$ and $d$ can be proved by showing the convexity of $g_{1}$ and $g_{2}$. The Hessian matrix of $g_{1}$ is: $H\left(g_{1}\right)=$ $\left[\begin{array}{cc}\frac{\partial^{2} g_{1}}{\partial P_{s}^{2}} & \frac{\partial^{2} g_{1}}{\partial P_{s} \partial d} \\ \frac{\partial^{2} g_{1}}{\partial d \partial P_{s}} & \frac{\partial^{2} g_{1}}{\partial d^{2}}\end{array}\right]=\left[\begin{array}{cc}\frac{\alpha_{1} \beta_{1}\left(\beta_{1}+1\right) d^{\beta_{1} l}}{a_{s}^{\beta_{1}} P_{s}^{\beta_{1}+2}} & -\frac{\alpha_{1} \beta_{1}^{2} l d^{\beta_{1} l-1}}{a_{s}^{\beta_{1}} P_{s}^{\beta_{1}+1}} \\ -\frac{\alpha_{1} \beta_{1}^{2} l d^{\beta_{1} l-1}}{a_{s}^{\beta_{1}} P_{s}^{\beta_{1}+1}} & \frac{\alpha_{1} \beta_{1} l\left(\beta_{1} l-1\right) d^{\beta_{1} l-2}}{\left(a_{s} P_{s}\right)^{\beta_{1}}}\end{array}\right]$ The determinant $\operatorname{det}\left[H\left(g_{1}\right)\right]=\frac{\alpha_{1}^{2} \beta_{1}^{2} l\left(\beta_{1}(l-1)-1\right) d^{2\left(\beta_{1} l-1\right)}}{a_{s}^{2 \beta_{1}} P_{s}^{2\left(\beta_{1}+1\right)}} \geq 0$ and $\frac{\partial^{2} g_{1}}{\partial P_{s}^{2}}, \frac{\partial^{2} g_{1}}{\partial d^{2}} \geq 0 \forall\left\{\left(P_{s}, d\right) \mid(d \geq 0) \wedge\left(P_{s} \geq 0\right) \wedge(l>1)\right\}$. This proves joint-convexity of $g_{1}$ in $P_{s}$ and $d$.

Similarly, on computing Hessian of $g_{2}$ to prove its convexity, we observe that $\frac{\partial^{2} g_{2}}{\partial P_{s}^{2}}=\frac{\alpha_{2} \beta_{2}\left(\beta_{2}+1\right)\left(a_{r} \eta\right)^{2}\left[\eta\left(\frac{D}{\epsilon}-d\right)^{l}\right]^{\beta_{2}}}{\left[a_{r} \eta\left(P_{T}-P_{s}\right)-\zeta_{P}\left(\frac{D}{\epsilon}-d\right)^{l}\right]^{2 \beta_{2}}}, \frac{\partial^{2} g_{2}}{\partial d^{2}}=$
$\frac{\left(P_{T}-P_{s}\right)\left(\frac{D}{\epsilon}-d\right)^{\beta_{2} l-2}\left(a_{r} \eta\left(\beta_{2} l-1\right)\left(P_{T}-P_{s}\right)+\zeta_{P}(l+1)\left(\frac{D}{\epsilon}-d\right)^{l}\right)}{\left[\alpha_{2} \beta_{2} \eta^{\beta_{2}+1} l a_{r}\right]^{-1}\left[a_{r} \eta\left(P_{T}-P_{s}\right)-\zeta_{P}\left(\frac{D}{\epsilon}-d\right)^{l}\right]^{\beta_{2}+2}}$, and $\operatorname{det}\left[H\left(g_{2}\right)\right]=\left[\frac{a_{r} \eta\left(\beta_{2}(l-1)-1\right)\left(P_{T}-P_{s}\right)+\zeta_{P} l\left(\frac{D}{\epsilon}-d\right)^{l}}{\left(a_{r} \eta\left(P_{T}-P_{s}\right)-\zeta_{P}\left(\frac{D}{\epsilon}-d\right)^{l}\right)^{3}}\left(\alpha_{2} \beta_{2} a_{r}\right)^{2}\right.$ $\left.\times \eta^{2\left(\beta_{2}+1\right)} l\left(\frac{D}{\epsilon}-d\right)^{2\left(\beta_{2}-1\right)}\right] \geq 0 \forall\left\{\left(P_{s}, d\right) \mid\left(d \leq \frac{D}{\epsilon}\right) \wedge\left(P_{s} \leq\right.\right.$ $\left.\left.P_{T}\right) \wedge(l \geq 2)\right\}$. This implies convexity of $\widehat{\text { out }_{1 J}}$ in $P_{s}$ and $d$.

\section{APPENDIX E}

\section{Proof of Joint Convexity of Constraint $C 8$}

In this appendix, we prove the joint convexity of $g_{C 8}=$ $\frac{\zeta_{P}\left(\frac{D}{\epsilon}-d\right)^{l}}{\eta a_{r}\left(P_{T}-P_{s}\right)}-1$ in $P_{s}$ and $d$. The determinant of the Hessian matrix of $g_{C 8}$ is $\operatorname{det}\left[H\left(g_{C 8}\right)\right]=\frac{\zeta_{P}^{2}(l-2) l\left(\frac{D}{\epsilon}-d\right)^{2(l-1)}}{\eta^{2} a_{r}^{2}\left(P_{T}-P_{s}\right)^{4}} \geq$ $0 \forall\left\{\left(P_{s}, d\right) \mid\left(d \leq \frac{D}{\epsilon}\right) \wedge\left(P_{s} \leq P_{T}\right) \wedge(l \geq 2)\right\}$. This along with non-negativity of $\frac{\partial^{2} g_{C 8}}{\partial P_{s}^{2}}=\frac{2 \zeta_{P}\left(\frac{D}{\epsilon}-d\right)^{l}}{\eta a_{r}\left(P_{T}-P_{s}\right)^{3}}$ and $\frac{\partial^{2} g_{C 8}}{\partial d^{2}}=$ $\frac{\zeta_{P}(l-1) l\left(\frac{D}{\epsilon}-d\right)^{l-2}}{\eta a_{r}\left(P_{T}-P_{s}\right)}$ for $P_{s} \leq P_{T}, d \leq \frac{D}{\epsilon}$, and $l \geq 1$ implies joint-convexity of $g_{C 8}$ in $P_{s}$ and $d$.

\section{REFERENCES}

[1] M. Hasna and M.-S. Alouini, "Optimal power allocation for relayed transmissions over rayleigh-fading channels," IEEE Trans. Wireless Commun., vol. 3, no. 6, pp. 1999-2004, Nov. 2004.

[2] S. Ikki, M. Uysal, and M. Ahmed, "Joint optimization of power allocation and relay location for decode-and-forward dual-hop systems over Nakagami-m fading channels," in Proc. IEEE GLOBECOM, Honolulu, HI, USA, Nov. 2009.

[3] S. Ikki and S. Aissa, "A study of optimization problem for amplify-andforward relaying over weibull fading channels with multiple antennas," IEEE Commun. Lett., vol. 15, no. 11, pp. 1148-1151, Nov. 2011.

[4] M. Wang and Z. Zhong, "Optimal power allocation and relay location for decode-and-forward dual-hop systems over weibull fading channels," in Proc. IWCMC, Limassol, Cyprus, Aug. 2012, pp. 240-244.

[5] C. Kundu and R. Bose, "Joint optimal power allocation and relay location for amplify-and-forward multihop relaying over lognormal channel," in Proc. IEEE VTC Spring, Dresden, Germany, June 2013.

[6] R. Cao and L. Yang, "The affecting factors in resource optimization for cooperative communications: A case study," IEEE Trans. Wireless Commun., vol. 11, no. 12, pp. 4351-4361, Dec. 2012.

[7] I. Kim and D. Kim, "Outage-constrained source-sum-power minimization in multiple-sensor single-DF-relay networks with direct links," in Proc. IEEE Int. Conf. on Ubiquitous and Future Netw. (ICUFN), Shanghai, 2014, pp. 113-114.

[8] I. Kim and D. Kim, "Outage-constrained source-sum-power minimization in multiple-sensor single-DF-relay networks," IEEE Commun. Lett., vol. 17, no. 7, pp. 1388-1391, July 2013.

[9] L. Varshney, "Transporting information and energy simultaneously," in Proc. IEEE Int. Symp. Inf. Theory (ISIT), Toronto, Canada, July 2008, pp. $1612-1616$.

[10] P. Grover and A. Sahai, "Shannon meets Tesla: Wireless information and power transfer," in Proc. IEEE Int. Symp. Inf. Theory (ISIT), Austin, TX, Jun. 2010, pp. 2363-2367.

[11] R. Zhang and C. K. Ho, "MIMO broadcasting for simultaneous wireless information and power transfer," IEEE Trans. Wireless Commun., vol. 12, no. 5, pp. 1989-2001, May 2013.

[12] A. Nasir, X. Zhou, S. Durrani, and R. Kennedy, "Relaying protocols for wireless energy harvesting and information processing," IEEE Trans. Wireless Commun., vol. 12, no. 7, pp. 3622-3636, July 2013.

[13] C. Zhong, H. Suraweera, G. Zheng, I. Krikidis, and Z. Zhang, "Wireless information and power transfer with full duplex relaying," IEEE Trans. Commun., vol. 62, no. 10, pp. 3447-3461, Oct. 2014.

[14] Z. Ding, S. Perlaza, I. Esnaola, and H. Poor, "Power allocation strategies in energy harvesting wireless cooperative networks," IEEE Trans. Wireless Commun., vol. 13, no. 2, pp. 846-860, Feb. 2014. 
[15] G. Zhu, C. Zhong, H. Suraweera, G. Karagiannidis, Z. Zhang, and T. Tsiftsis, "Wireless information and power transfer in relay systems with multiple antennas and interference," IEEE Trans. Commun., vol. 63, no. 4, pp. 1400-1418, Apr. 2015.

[16] I. Krikidis, "Simultaneous information and energy transfer in large-scale networks with/without relaying," IEEE Trans. Commun., vol. 62, no. 3, pp. 900-912, Mar. 2014.

[17] Z. Ding, I. Krikidis, B. Sharif, and H. Poor, "Wireless information and power transfer in cooperative networks with spatially random relays," IEEE Trans. Wireless Commun., vol. 13, no. 8, pp. 4440-4453, Aug. 2014.

[18] D. Michalopoulos, H. Suraweera, and R. Schober, "Relay selection for simultaneous information transmission and wireless energy transfer: A tradeoff perspective," IEEE J. Sel. Areas Commun., vol. 33, no. 8, pp. 1578-1594, Aug 2015.

[19] Z. Ding, C. Zhong, D. Ng, M. Peng, H. Suraweera, R. Schober, and H. Poor, "Application of smart antenna technologies in simultaneous wireless information and power transfer," IEEE Commun. Mag., vol. 53, no. 4, pp. 86-93, Apr. 2015.

[20] L. Liu, R. Zhang, and K.-C. Chua, "Wireless information and power transfer: A dynamic power splitting approach," IEEE Trans. Commun., vol. 61, no. 9, pp. 3990-4001, September 2013.

[21] H. Suraweera, G. Karagiannidis, and P. Smith, "Performance analysis of the dual-hop asymmetric fading channel," IEEE Trans. Wireless Commun., vol. 8, no. 6, pp. 2783-2788, June 2009.

[22] S. De and R. Singhal, "Toward uninterrupted operation of wireless sensor networks," IEEE Computer Mag., vol. 45, no. 9, pp. 24-30, Sep. 2012

[23] D. Mishra, K. Kaushik, S. De, S. Basagni, K. Chowdhury, S. Jana, and W. Heinzelman, "Implementation of multi-path energy routing," in Proc. IEEE PIMRC, Washington D.C., USA, Sep. 2014.

[24] K. Kaushik, D. Mishra, S. De, S. Basagni, W. Heinzelman, K. Chowdhury, and S. Jana, "Experimental demonstration of multi-hop RF energy transfer," in Proc. IEEE PIMRC, London, UK, Sep. 2013, pp. 538-542.

[25] M. Yu, J. Li, and H. Sadjadpour, "Amplify-forward and decode-forward: The impact of location and capacity contour," in Proc. IEEE Military Commun. Conf. (MILCOM), Atlantic City, NJ, Oct. 2005, pp. 1609-1615 Vol. 3.

[26] M. K. Simon and M.-S. Alouini, Digital communication over fading channels, 2nd ed. New York, USA: Wiley, 2005.

[27] C. A. Balanis, Antenna theory: analysis and design. John Wiley \& Sons, 2012.

[28] D. Mishra, S. De, S. Jana, S. Basagni, K. Chowdhury, and W. Heinzelman, "Smart RF energy harvesting communications: Challenges and opportunities," IEEE Commun. Mag., vol. 53, no. 4, pp. 70-78, Apr. 2015.

[29] D. Mishra and S. De, "Optimal relay placement in two-hop RF energy transfer," IEEE Trans. Commun., vol. 63, no. 5, pp. 1635-1647, May 2015.

[30] FCC codes of regulation, part 15. [Online]. Available: http://www.access.gpo.gov/nara/cfr/waisidx03/

[31] J. Laneman, D. Tse, and G. W. Wornell, "Cooperative diversity in wireless networks: Efficient protocols and outage behavior," IEEE Trans. Inf. Theory, vol. 50, no. 12, pp. 3062-3080, Dec. 2004.

[32] M. Hasna and M.-S. Alouini, "End-to-end performance of transmission systems with relays over rayleigh-fading channels," IEEE Trans. Wireless Commun., vol. 2, no. 6, pp. 1126-1131, Nov. 2003.

[33] M. Bocus, C. Dettmann, and J. Coon, "An approximation of the first order Marcum Q-function with application to network connectivity analysis," IEEE Commun. Lett., vol. 17, no. 3, pp. 499-502, Mar. 2013.

[34] R. Morsi, D. Michalopoulos, and R. Schober, "Multiuser scheduling schemes for simultaneous wireless information and power transfer over fading channels," IEEE Trans. Wireless Commun., vol. 14, no. 4, pp. 1967-1982, Apr. 2015.

[35] A. Castano-Martinez and F. Lopez-Blazquez, "Distribution of a sum of weighted noncentral chi-square variables," TEST, vol. 14, no. 2, pp. 397-415, Dec. 2005.

[36] M. S. Bazaraa, H. D. Sherali, and C. M. Shetty, Nonlinear Programming: Theory and Applications. New York: John Wiley and Sons, 2006.

[37] A. Cambini and L. Martein, Generalized convexity and optimization: Theory and applications. Springer, 2008, vol. 616.

[38] M. J. Powell, "Convergence properties of algorithms for nonlinear optimization," Siam Review, vol. 28, no. 4, pp. 487-500, Dec. 1986.

[39] A. D. Belegundu and T. R. Chandrupatla, Optimization concepts and applications in engineering. Cambridge University Press, 2011.

[40] I. Csiszár and G. Tusnády, "Information geometry and alternating minimization procedures," Statistics and Decisions, Supplement Issue, vol. 1, no. 1, pp. 205-237, 1984.
[41] Y. Sun, . Baricz, and S. Zhou, "On the monotonicity, log-concavity, and tight bounds of the generalized Marcum and Nuttall Q-functions," IEEE Trans. Inf. Theory, vol. 56, no. 3, pp. 1166-1186, Mar. 2010.

[42] S. Boyd and L. Vandenberghe, Convex optimization. Cambridge university press, 2004.

[43] M. Avriel, D. E., S. Schaible, and I. Zang, Generalized concavity. Siam, 2010, vol. 63

[44] A. Prékopa, "Logarithmic concave measures and related topics," in Stochastic programming. Citeseer, 1980

[45] M. Bagnoli and T. Bergstrom, "Log-concave probability and its applications," Economic theory, vol. 26, no. 2, pp. 445-469, 2005.

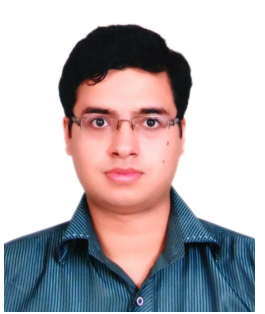

Deepak Mishra (S'13) received his B.Tech degree in Electronics and Communication Engineering from Guru Gobind Singh Indraprastha University, Delhi, India, in 2012. He is currently pursuing the Ph.D. degree from the Department of Electrical Engineering, IIT Delhi, India. His research interests include RF energy harvesting cooperative communication networks and energy optimization schemes for uninterrupted operation of mobile ad hoc networks.

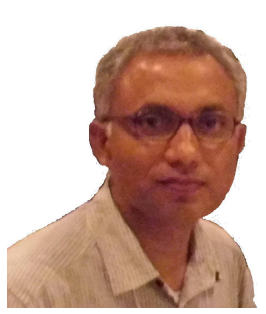

Swades De (S'02-M'04-SM'14) received the Ph.D. degree from the State University of New York at Buffalo, NY, USA, in 2004. He is currently an Associate Professor in the Department of Electrical Engineering, IIT Delhi, India. In 2004, he worked as an ERCIM researcher at ISTI-CNR, Italy. From 2004 to 2006 he was with NJIT as an Assistant Professor. His research interests include performance study, resource efficiency in wireless networks, broadband wireless access, and optical communication systems.

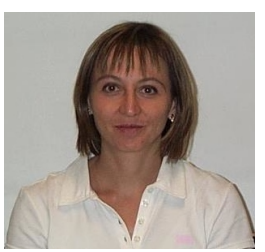

Carla-Fabiana Chiasserini (M'98-SM'09) received her Ph.D. in 2000 from Politecnico di Torino, where she is currently an Associate Professor. From 1998 to 2003, she has been a vising researcher at UCSD. She has been a visiting professor at Monash University in 2012. She is an Associate Researcher at CNRIEIIT. Her research interests include protocols and performance analysis of wireless networks. Dr. Chiasserini has over 250 publications at major venues. 